\title{
A multithickness sea ice model accounting for sliding friction
}

Article

Published Version

Wilchinsky, A. V., Feltham, D. L. and Miller, P. A. (2006) A multithickness sea ice model accounting for sliding friction. Journal of Physical Oceanography, 36 (9). pp. 1719-1738. ISSN 0022-3670 doi: https://doi.org/10.1175/JPO2937.1 Available at https://centaur.reading.ac.uk/34910/

It is advisable to refer to the publisher's version if you intend to cite from the work. See Guidance on citing.

Published version at: http://dx.doi.org/10.1175/JPO2937.1

To link to this article DOI: http://dx.doi.org/10.1175/JPO2937.1

Publisher: American Meteorological Society

All outputs in CentAUR are protected by Intellectual Property Rights law, including copyright law. Copyright and IPR is retained by the creators or other copyright holders. Terms and conditions for use of this material are defined in the End User Agreement.

\section{www.reading.ac.uk/centaur}

\section{CentAUR}

Central Archive at the University of Reading

Reading's research outputs online 


\title{
A Multithickness Sea Ice Model Accounting for Sliding Friction
}

\author{
Alexander V. Wilchinsky,* Daniel L. Feltham, ${ }^{+}$and Paul A. Miller ${ }^{\#}$ \\ Centre for Polar Observation and Modelling, Department of Earth Sciences, University College London, London, United Kingdom
}

(Manuscript received 7 September 2005, in final form 2 February 2006)

\begin{abstract}
A multithickness sea ice model explicitly accounting for the ridging and sliding friction contributions to sea ice stress is developed. Both ridging and sliding contributions depend on the deformation type through functions adopted from the Ukita and Moritz kinematic model of floe interaction. In contrast to most previous work, the ice strength of a uniform ice sheet of constant ice thickness is taken to be proportional to the ice thickness raised to the $3 / 2$ power, as is revealed in discrete element simulations by Hopkins. The new multithickness sea ice model for sea ice stress has been implemented into the Los Alamos "CICE" sea ice model code and is shown to improve agreement between model predictions and observed spatial distribution of sea ice thickness in the Arctic.
\end{abstract}

\section{Introduction}

Sea ice models describe melting and freezing (thermodynamics) and movement and deformation of the ice cover (dynamics) in response to atmospheric and oceanic forces. Model simulations reveal the importance of sea ice dynamics to the climate system. For example, in comparing simulations of Arctic sea ice volume with submarine gathered data, Hilmer and Lemke (2000) concluded that better models of sea ice dynamics are required to improve understanding of the interannual variability and discern the true climate signal. In this paper, we focus on sea ice stresses resulting from deformation of the ice cover.

The sea ice cover is formed from floes that are of approximately convex polygonal shape, have lateral dimension of between $100 \mathrm{~m}$ and $5 \mathrm{~km}$, and are several meters thick. Sea ice deformation occurs through the

\footnotetext{
* Additional affiliation: Institute of Mathematics and Mechanics, Kazan State University, Kazan, Republic of Tatarstan, Russia.

+ Additional affiliation: British Antarctic Survey, Cambridge, United Kingdom.

\# Additional affiliation: Department of Physical Geography and Ecosystems Analysis, Ecosystem Modelling and Biodiversity Studies (EMBERS) Group, Lund University, Lund, Sweden.
}

Corresponding author address: Alexander V. Wilchinsky, Centre for Polar Observation and Modelling, Dept. of Earth Sciences, University College London, Gower Street, London, WC1E 6BT, United Kingdom.

E-mail: aw@cpom.ucl.ac.uk relative motion of sea ice floes: under local divergence, sea ice floes can separate to form linear regions of open water called leads; in local shear, floes can slide along their common edges; and in local convergence, floes can break up to form linear regions of rubble piled above and below the ice cover along floe edges known as pressure ridges. Typically floe-scale deformation involves ridging and sliding or opening and sliding, but all types of deformation are usually present in any region containing a collection of floes.

During pressure ridging, the ice cover first breaks in flexure into blocks and the ridging stress is determined by the work required to move the ice blocks against gravity and ridging friction to form a pressure ridge. When floes slide past each other, the sliding stress is determined by friction between the floe edges. The frictional stress is normally taken to be independent of the rate of deformation so that the cumulative sea ice stress is independent of strain-rate magnitude. Since the work done in forming pressure ridges or sliding of floes past each other is irreversible, sea ice rheological behavior is considered to be plastic.

Since ridging redistributes ice among different thicknesses whereas sliding does not, the redistribution of ice thickness depends upon the relative amount of sea ice deformation realized through ridging as compared with sliding (and opening). The resultant sea ice stress used in a model of sea ice dynamics also depends upon the relative amounts of ridging, sliding, and opening as the stresses involved in these types of deformation differ. The relative amounts of ridging and sliding depend 
upon the type of deformation of the ice cover; for example, more sliding of floes past each other occurs in pure shear than in pure divergence.

Most sophisticated sea ice codes treat the ice within a grid cell to be distributed between several thickness categories and implement a parameterization for the ridging work fraction introduced by Flato and Hibler (1995). A particular feature of this parameterization is that it implies the absence of sliding friction in pure convergence, while discrete simulations (Hopkins 1996; Ukita and Moritz 2000) show that the irregular shape of floes leads to significant interfloe sliding in this case.

Sea ice models require an expression relating sea ice strength (maximum stress in compression) to the thickness of the ice that is being deformed. Most sea ice models either set the ice strength to be proportional to the potential energy change in forming a pressure ridge, in which case the strength is proportional to ice thickness squared, or treat the strength to be proportional to ice thickness, adopting a parameterization due to Hibler (1979). However, sophisticated discrete element simulations of the pressure ridging process (Hopkins 1998) suggest that the ridging force is actually proportional to the thickness of the ice being ridged to the $3 / 2$ power.

In this paper, we introduce a new model of sea ice stress and thickness redistribution that realistically treats the relative fraction of ridging to sliding deformation and treats ridging stresses as being proportional to the thickness of the ice being ridged to the $3 / 2$ power.

The paper is divided as follows: In section 2, we briefly summarize classical thickness distribution theory before presenting equations for the ridging, sliding, and opening strengths of a nonuniform sea ice cover. In section 3, we present the theory for the construction of a yield curve using the principle of minimization of maximum shear stress for a nonuniform sea ice cover. The specific functional forms for the dependence of ridging and sliding strength upon ice thickness, and examples of how they affect the yield curve, are discussed in section 4 . In section 5 , we describe the inclusion of our new physics into the Los Alamos "CICE" sea ice model code and present simulation results that suggest the new model of sea ice stress and thickness redistribution more accurately accounts for the observed variation of ice thickness across the Arctic Ocean. In section 6, we present a summary of our work and our main conclusions.

\section{Sea ice redistribution and strength of a nonuniform sea ice cover}

Before we begin our development, it is useful to define some common notation that will be used through- out. We denote tensors by boldface symbols and define the first and second invariants of the two-dimensional, horizontal stress and strain-rate tensors $\boldsymbol{\sigma}$ and $\dot{\boldsymbol{\epsilon}}$ through their principal values, the first of which is the largest,

$$
\sigma_{\mathrm{I}}=\frac{1}{2}\left(\sigma_{1}+\sigma_{2}\right)=\frac{1}{2} \sigma_{i i}, \quad \sigma_{\mathrm{II}}=\frac{1}{2}\left(\sigma_{1}-\sigma_{2}\right)=\sqrt{\frac{1}{2} \sigma_{i j}^{*} \sigma_{i j}^{*}},
$$

$\dot{\epsilon}_{1}=\dot{\epsilon}_{1}+\dot{\epsilon}_{2}=\dot{\epsilon}_{i i}, \quad \dot{\epsilon}_{\mathrm{II}}=\dot{\epsilon}_{1}-\dot{\epsilon}_{2}=\sqrt{2 \dot{\epsilon}_{i j}^{*} \dot{\epsilon}_{i j}^{*}}$,

where an asterisk denotes the traceless part of a tensor; for example, $\boldsymbol{\sigma}^{*}=-\sigma_{I} \mathbf{1}+\boldsymbol{\sigma}$. An alternative, useful pair of strain rate invariants are

$$
|\dot{\epsilon}|=\sqrt{\dot{\epsilon}_{\mathrm{I}}^{2}+\dot{\epsilon}_{\mathrm{II}}^{2}}, \quad \theta=\arctan \left(\frac{\dot{\epsilon}_{\mathrm{II}}}{\dot{\epsilon}_{\mathrm{I}}}\right),
$$

where $\theta$, being a measure of the ratio of shear to divergence, defines the strain rate type, and

$$
\dot{\epsilon}_{\mathrm{I}}=|\dot{\epsilon}| \cos \theta, \quad \dot{\epsilon}_{\mathrm{II}}=|\dot{\epsilon}| \sin \theta .
$$

Throughout the paper we will refer to a yield-curve aspect ratio as the ratio of its maximum shear stress to the maximum pressure, which is normally less than unity. The standard elliptic yield curve used by Hibler (1979) would have an aspect ratio of 1/2.

\section{a. Evolution of sea ice thickness distribution}

We briefly recapitulate the essential elements of the classical theory of ice thickness distribution (Thorndike et al. 1975), which is necessary for our further development. If ice type is identified by its thickness $h$ (normalized by $1 \mathrm{~m}$ ) only, the state of the sea ice cover can be described by the ice thickness distribution function $g(h)$, defined such that the fractional area of ice with thickness in the range $h$ to $h+d h$ is given by $g(h) d h$. Under pure convergence of a unit area of sea ice, during a time $\delta t$, only the fractional area $A_{r}(\delta t)$ is broken up to form ridges (consisting of sails and keels). The ridging participation function $\gamma_{r}(h)$ [where $\max \left(\gamma_{r}\right)=$ $\left.\gamma_{r}(0)=1\right]$ is the fraction of ice of thickness $h$ that ridges so that $\gamma_{r}(h) g(h)$ is the nonnormalized distribution of ice in $A_{r}$ and the normalized distribution is

$$
a_{r}(h)=\frac{\gamma_{r}(h) g(h)}{\int_{0}^{\infty} \gamma_{r}\left(h^{\prime}\right) g\left(h^{\prime}\right) d h^{\prime}} .
$$

The ridging participation function reflects how the plastic deformation work done during a deformation of the ice cover is minimized by distributing the deformation among different ice thicknesses for a particular sea ice state: Although the work done in a deformation of the ice cover is a minimum if only the thinnest ice deforms, 
this is usually not possible without also deforming the surrounding thicker ice. The standard choice of the ridging participation function is

$$
\begin{aligned}
& \gamma_{r}=\max \left[\left(1-\frac{G(h)}{C_{1}}\right), 0\right] \quad G(h)=\int_{0}^{h} g\left(h^{\prime}\right) d h^{\prime}, \\
& G<C_{1}
\end{aligned}
$$

(Thorndike et al. 1975), where only the thinnest fraction $C_{1}$ of the ice cover is ridged (typically $C_{1}$ is set to $0.15)$. The proportion of the ice ridged within the fraction $C_{1}$ gradually decreases from 1 to 0 as the thickness approaches the thickest ice available for ridging. Adopting the above participation function, the normalized distribution function of ice participated in ridging, (5), becomes

$$
a_{r}(h)=\max \left\{\frac{2 g(h)}{C_{1}}\left[1-\frac{G(h)}{C_{1}}\right], 0\right\}, \quad C_{1}=G\left(h^{*}\right),
$$

where $h^{*}$ is the upper limit of the ice thickness affected by ridging.

The transfer function $\beta\left(h_{1}, h_{2}\right)$ defines the area of ice of thickness $h_{2}$ produced by ridging of a unit area of ice of uniform thickness $h_{1}$. Thus the area of deformed ice produced after ridging of a unit area of thickness $h_{1}$ is $\int_{0}^{\infty} \beta\left(h_{1}, h_{2}\right) d h_{2}$ and the total deformation (fractional decrease in area) is $\int_{0}^{\infty} \beta\left(h_{1}, h_{2}\right) d h_{2}-1$.

The function $\psi$ is defined to be the rate of change of area of ice of thickness $h$ within a nonuniform sea ice cover per unit convergent deformation, so the change of area of ice of thickness $h$ during time $\delta t$ in a unit area of sea ice is

$$
\delta t \psi(h)\left|\dot{\epsilon}_{I}\right|=A_{r}\left[-a_{r}(h)+\int_{0}^{\infty} \beta\left(h^{\prime}, h\right) a_{r}\left(h^{\prime}\right) d h^{\prime}\right] .
$$

The fact that all redistribution of ice thickness in pure convergence is realized through ridging is expressed by

$$
\int_{0}^{\infty} \psi(h) d h=-1
$$

so, integrating (8), the sea ice area undergoing ridging deformation is seen to be

$$
A_{r}=\left|\dot{\epsilon}_{I}\right| \delta t\left\{\int_{0}^{\infty}\left[a_{r}(h)-\int_{0}^{\infty} \beta\left(h^{\prime}, h^{\prime \prime}\right) a_{r}\left(h^{\prime}\right) d h^{\prime}\right] d h^{\prime \prime}\right\}^{-1} .
$$

Elimination of $A_{r}$ from (8) and (10) determines the change of area of ice of thickness $h$ per unit deformation in pure convergence as

$$
\psi(h)=\frac{-a_{r}(h)+\int_{0}^{\infty} \beta\left(h^{\prime}, h\right) a_{r}\left(h^{\prime}\right) d h^{\prime}}{1-\int_{0}^{\infty} \int_{0}^{\infty} \beta\left(h^{\prime}, h^{\prime \prime}\right) a_{r}\left(h^{\prime}\right) d h^{\prime} d h^{\prime \prime}} .
$$

The evolution of the ice thickness distribution is given by

$$
\frac{d g}{d t}=\Psi+F_{l}-g\left(\dot{\epsilon}_{I}+\frac{\partial f}{\partial h}\right)
$$

(Thorndike et al. 1975), where $d / d t$ is the material time derivative, $\Psi$ is the ridging redistribution function, $f$ is the vertical freeze/melt rate, and $F_{l}$ is the lateral freeze/ melt rate (added by Hibler 1980). The sea ice redistribution function $\Psi$ accounts for the amount of ridging and opening as a function of the deformation,

$$
\Psi=|\dot{\epsilon}|\left[\alpha_{r}(\theta) \psi+\chi(h) \alpha_{o}(\theta)\right],
$$

where $\alpha_{r}(\theta)$ and $\alpha_{o}(\theta)$ describe the relative amount of deformation realized through pure convergence-type ridging and pure divergence-type opening, respectively. In other words, $\alpha_{r}(\theta)$ is the fraction of ice area lost during a unit deformation of a particular type described by $\theta$. This is equal to unity when pure convergence is considered, $\theta=\pi$. Similarly, $\alpha_{o}(\theta)$ is the fraction of open water area created during a unit deformation of a particular type described by $\theta$, which is unity for pure divergence, $\theta=0$. The function $\chi(h)$ is 2 times the Dirac delta function, so defined that $\chi(h)=0, \forall h>0$, and $\int_{0}^{\infty} \chi(h) d h=1$. As $\Psi$ describes only mechanical ice redistribution, by neglecting all thermodynamics effects and integrating (12) over all ice thicknesses we obtain

$$
\int_{0}^{\infty} \Psi(h) d h=\dot{\epsilon}_{I}
$$

Similar integration of (13) and accounting for (4), (9), and (14) produces the standard relationship between the opening and ridging modes (Thorndike et al. 1975),

$$
\alpha_{0}=\alpha_{r}+\cos \theta
$$

The particular form of $\alpha_{r}$ used in this work will be discussed later on.

\section{b. Ridging, sliding, and opening ice strengths of a nonuniform sea ice cover}

During sea ice deformation ridging, sliding, and opening occur simultaneously. Their contributions vary with the deformation type: for example, more sliding occurs in shear than in compression. The importance of a particular type of interfloe motion on the work done is described by its strength. The ridging ice strength $P_{r}$ can be defined as the work necessary to produce ridges in a unit convergence of a unit area of sea ice. Since 
ridging of an ice cover with a range of ice thicknesses is not uniformly distributed over all thicknesses, the ridging ice strength $P_{r}$ is given by the integral of the ridging strengths of single ice thicknesses $P_{r}^{*}(h)$ weighted by their fractional areas undergoing ridging:

$$
\begin{aligned}
P_{r} & =\frac{A_{r}}{\dot{\epsilon}_{\mathrm{I}} \delta t} \int_{0}^{\infty} P_{r}^{*}(h) a_{r}(h) d h \\
& =\frac{\int_{0}^{\infty} P_{r}^{*}(h) a_{r}(h) d h}{1-\int_{0}^{\infty} \int_{0}^{\infty} \beta\left(h^{\prime}, h^{\prime \prime}\right) a_{r}\left(h^{\prime}\right) d h^{\prime} d h^{\prime \prime}},
\end{aligned}
$$

where the final expression was obtained using (10). With the assumption that no lead opening occurs in pure convergence, the single-layer ice strength, $P_{r}^{*}(h)$, is given by the work necessary to ridge a unit area of ice into thicknesses $h^{\prime}$ areally distributed as $\beta\left(h, h^{\prime}\right)$ divided by the convergence magnitude, $1-\int_{0}^{\infty} \beta\left(h, h^{\prime}\right) d h^{\prime}$.

The sliding ice strength $P_{s}$ of a nonuniform ice cover can be treated in a similar fashion to that in ridging. Let us denote by $P_{s}^{*}(h)$ the work done by frictional sliding along floe-floe boundaries in a unit sliding deformation of ice of thickness $h$. Sliding deformation rate $|\dot{d}|$ is defined as the sliding velocity integrated over all floe edges in a unit sea ice area (Moritz and Ukita 2000). For a fixed deformation rate $|\dot{\epsilon}|$ the sliding rate changes as the flow type changes. This is described by introduction of the sliding mode $\alpha_{s}(\theta)$ such that $|\dot{d}|=\alpha_{s}(\theta)|\dot{\epsilon}|$. Sliding work increases with the floe thickness, and thus it is natural to introduce a sliding participation function, analogous to the ridging participation function, that models the preferential sliding of thinner ice floes in shear, $\gamma_{s}(h)\left[\right.$ where $\left.\max \left(\gamma_{s}\right)=\gamma_{s}(0)=1\right]$. The relation between $\gamma_{r}$ and $\gamma_{s}$ depends on the relation between the ridging and sliding ice strengths: If the ridging and sliding ice strengths are proportional, then one would expect that $\gamma_{r} \approx \gamma_{s}$, whereas if the sliding ice strength increases less rapidly with ice thickness than the ridging strength does, one would expect there to be more thicker ice involved in sliding than in ridging. The fraction of ice area undergoing sliding deformation is given by $A_{s}=\int_{0}^{\infty} \gamma_{s}(h) g(h) d h$, and the normalized ice thickness distribution is

$$
a_{s}(h)=\frac{\gamma_{s}(h) g(h)}{\int_{0}^{\infty} \gamma_{s}\left(h^{\prime}\right) g\left(h^{\prime}\right) d h^{\prime}} .
$$

As no sliding occurs out of this active area, the sliding rate in it is $\left|\dot{d}^{s}\right|=|\dot{d}| / A_{s}$. The cumulative work of sliding per unit sliding deformation is given by

$$
P_{s}=\frac{1}{|\dot{d}|} \int_{0}^{\infty} P_{s}^{*}(h)\left|\dot{d}^{s}\right| A_{s} a_{s}(h) d h=\int_{0}^{\infty} P_{s}^{*}(h) a_{s}(h) d h .
$$

Note that the same result is obtained if $\gamma_{s}(h)$ describes not the difference in the ice fraction undergoing sliding, but the difference of the sliding-rate magnitude between different ice thicknesses when the entire sea ice area undergoes sliding. In this case $a_{s}(h)$ would describe the relative distribution of sliding among different ice thicknesses and the last integral in (18) would determine the cumulative work arising through it.

We can treat ice strength in opening $P_{o}$ of a nonuniform ice cover in the same way as strength in ridging and sliding. The work performed in a unit pure divergence deformation of an ice cover of a uniform thickness $h$ is defined to be $P_{o}^{*}(h)$, which describes the contribution of tensile stress due to lead formation. We introduce an opening participation function $\gamma_{o}(h)$ [where $\max \left(\gamma_{o}\right)=\gamma_{o}(0)=1$ ] so that the fractional area undergoing the opening deformation is $A_{o}=\int_{0}^{\infty}$ $\gamma_{o}(h) g(h) d h$, and the mean strain rate in this region is $\dot{\epsilon}_{I} / A_{o}$. The normalized opening ice thickness distribution is

$$
a_{o}(h)=\frac{\gamma_{o}(h) g(h)}{\int_{0}^{\infty} \gamma_{o}\left(h^{\prime}\right) g\left(h^{\prime}\right) d h^{\prime}} .
$$

The cumulative work per unit deformation under divergence due to sea ice opening then takes the form

$$
P_{o}=\int_{0}^{\infty} P_{o}^{*}(h) a_{o}(h) d h .
$$

\section{Sea ice yield curve from the deformation power of a nonuniform layer of sea ice}

\section{a. Underlying theory for a uniform sea ice layer}

Rothrock (1975) related the rate of doing plastic work by deformation of a unit-thickness sea ice cover to the work done by ridging. Ukita and Moritz (1995, 2000) and Moritz and Ukita (2000, hereinafter UM), generalized this approach by including the contribution to plastic work from sliding so that the rate of doing plastic work in deformation is given by

$$
\sigma_{\mathrm{I}} \dot{\epsilon}_{\mathrm{I}}+\sigma_{\mathrm{II}} \dot{\epsilon}_{\mathrm{II}}=|\dot{\epsilon}|\left[P_{r}^{*} \alpha_{r}(\theta)+P_{s}^{*} \alpha_{s}(\theta)\right],
$$

where the stress and strain-rate principal axes are aligned. The ridging and sliding modes, $\alpha_{r}$ and $\alpha_{s}$, determine how much ridging and sliding are produced during sea ice deformation. Moritz and Ukita (2000) determined the ridging and sliding modes using kine- 


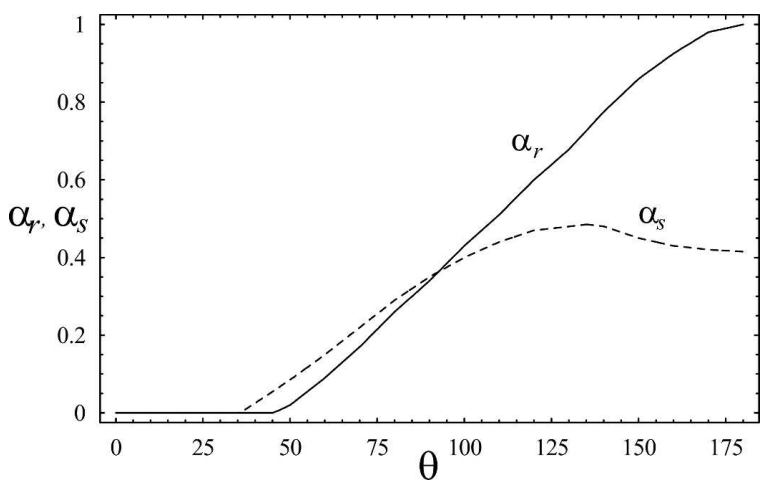

FIG. 1. Ridging mode $\alpha_{r}(\theta)$ (solid line) and sliding mode $\alpha_{s}(\theta)$ (dashed line) as found by Ukita and Moritz (2000) for a Poisson distribution of cracks.

matic considerations: The deformation of a region containing a collection of randomly oriented polygonal floes was interpolated and rotated along the floe edges in the region so that the deformation perpendicular to the floe edges (ridging/opening) and parallel to the floe edges (sliding) could be calculated. The ridging and sliding modes that we will be using throughout the paper, calculated by UM, are depicted in Fig. 1. (Note that $\theta=0$ in pure divergence, $\theta=\pi / 4$ in uniaxial extension, $\theta=\pi / 2$ in pure shear, $\theta=3 \pi / 4$ in uniaxial contraction, and $\theta=\pi$ for pure convergence.)

The plastic yield curve was derived by UM using an assumption that a value of $\sigma_{I}$ corresponds to such $\theta$ that minimizes the shear stress found from simple rearrangement of (21),

$$
\sigma_{\mathrm{II}}=\frac{1}{\sin \theta}\left[P_{r}^{*} \alpha_{r}(\theta)+P_{s}^{*} \alpha_{s}(\theta)-\sigma_{\mathrm{I}} \cos \theta\right]
$$

[where we have used (4)] so that the yield curve is given by the locus of points

$$
\left\{\sigma_{\mathrm{I}}, \min _{\theta} \sigma_{\mathrm{II}}\left(\sigma_{\mathrm{I}}, \theta\right)\right\}
$$

\section{b. Development of the theory for a nonuniform sea ice cover}

The ridging and sliding modes $\alpha_{r}$ and $\alpha_{s}$ determined by UM were found from kinematic considerations concerning floe geometry for an ice cover of uniform thickness. We adopt the same ridging and sliding modes for an ice cover consisting of ice of various thicknesses, as this does not affect the kinematic arguments used by UM. The participation functions account for the concentration of deformation into the thinner ice. The rate of doing plastic work per unit sea ice deformation of a nonuniform sea ice cover is similar to that given by (21) (work per unit time) divided by the strain rate magnitude $|\dot{\epsilon}|$,

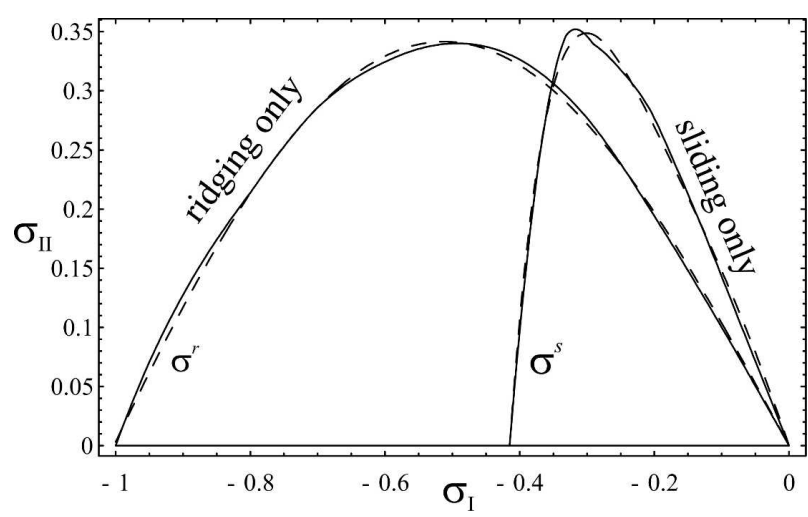

FIG. 2. The normalized ridging and sliding stress contributions $\boldsymbol{\sigma}^{r}$ and $\boldsymbol{\sigma}^{s}$ (solid) and their polynomial approximations (dashed).

$$
\sigma_{\mathrm{I}} \cos \theta+\sigma_{\mathrm{II}} \sin \theta=P_{r} \alpha_{r}(\theta)+P_{s} \alpha_{s}(\theta)+P_{o} \alpha_{o}(\theta),
$$

where we have added a term that accounts for work done in opening and used the multilayer ice strengths for ridging, sliding, and opening given by (16), (18), and (20), respectively.

In a similar manner to Wilchinsky and Feltham (2004), we consider ridging, sliding, and opening stress contributions normalized per unit ice strength separately and these are determined by

$$
\begin{array}{ll}
\sigma_{\mathrm{I}}^{r} \cos \theta+\sigma_{\mathrm{II}}^{r} \sin \theta=\alpha_{r}(\theta), & \sigma_{\mathrm{II}}^{r}=\min _{\theta} \sigma_{\mathrm{II}}^{r}\left(\sigma_{\mathrm{I}}^{r}, \theta\right), \\
\sigma_{\mathrm{I}}^{s} \cos \theta+\sigma_{\mathrm{II}}^{s} \sin \theta=\alpha_{s}(\theta), & \sigma_{\mathrm{II}}^{s}=\min _{\theta} \sigma_{\mathrm{II}}^{s}\left(\sigma_{\mathrm{I}}^{s}, \theta\right), \\
\sigma_{\mathrm{I}}^{o} \cos \theta+\sigma_{\mathrm{II}}^{o} \sin \theta=\alpha_{o}(\theta), & \sigma_{\mathrm{II}}^{o}=\min _{\theta} \sigma_{\mathrm{II}}^{o}\left(\sigma_{\mathrm{I}}^{o}, \theta\right) .
\end{array}
$$

The word "normalized" is used to accentuate their independence of the sea ice thickness. The solutions $\left\{\sigma_{\mathrm{I}}^{r}(\theta), \sigma_{\mathrm{II}}^{r}\left(\sigma_{\mathrm{I}}^{r}\right)\right\},\left\{\sigma_{\mathrm{I}}^{s}(\theta), \sigma_{\mathrm{II}}^{s}\left(\sigma_{\mathrm{I}}^{s}\right)\right\}$ have been calculated numerically, and polynomial approximations are given in the appendix. The yield curve contribution from ridging and sliding and their polynomial interpolations are presented in Fig. 2 using $\alpha_{r}$ and $\alpha_{s}$ calculated by UM and presented in Fig. 1. The direction of the strain rate for each of the yield curves is normal to the yield curve, departing from normality slightly on the left branch of the sliding contribution yield curve (which we believe is due to numerical errors in data approximation). We treat the flow law for the separate ridging and sliding yield curves to be normal. 
We introduce the tensor

$$
\boldsymbol{\sigma}^{a}(\dot{\boldsymbol{\epsilon}}) \equiv P_{r} \boldsymbol{\sigma}^{r}(\dot{\boldsymbol{\epsilon}})+P_{s} \boldsymbol{\sigma}^{s}(\dot{\boldsymbol{\epsilon}})+P_{o} \boldsymbol{\sigma}^{o}(\dot{\boldsymbol{\epsilon}}),
$$

where $\boldsymbol{\sigma}^{r}(\dot{\boldsymbol{\epsilon}}), \boldsymbol{\sigma}^{s}(\dot{\boldsymbol{\epsilon}})$ and $\boldsymbol{\sigma}^{o}(\dot{\boldsymbol{\epsilon}})$ are the normalized ridging, sliding, and opening stress tensors determined by (25)-(27). Since the principal axes of the stresses and strain rate coincide, we can write

$$
\sigma_{\mathrm{I}}^{a}=P_{r} \sigma_{\mathrm{I}}^{r}+P_{s} \sigma_{\mathrm{I}}^{s}+P_{o} \sigma_{\mathrm{I}}^{o}
$$

and (provided the largest principal values of $\boldsymbol{\sigma}^{r}, \boldsymbol{\sigma}^{s}$, and $\boldsymbol{\sigma}^{o}$ belong to the same axis)

$$
\sigma_{\mathrm{II}}^{a}=P_{r} \sigma_{\mathrm{II}}^{r}+P_{s} \sigma_{\mathrm{II}}^{s}+P_{o} \sigma_{\mathrm{II}}^{o}
$$

The definition of $\boldsymbol{\sigma}^{a}$ and (29) and (30) imply that $\boldsymbol{\sigma}^{a}$ satisfies (24). Furthermore, since $\sigma_{\mathrm{I}}^{a}(\theta)$ is a monotone function with $\sigma_{\mathrm{I}}^{a}(0)=0$ and $\sigma_{\mathrm{I}}^{a}(\pi)=\sigma_{\mathrm{I}}(\pi)<0$, for a particular value of $\sigma_{I}$ satisfying $\sigma_{\mathrm{I}}^{a}(\pi) \leq \sigma_{\mathrm{I}} \leq 0$, we can find $\theta_{0}$ such that $\sigma_{\mathrm{I}}=\sigma_{\mathrm{I}}^{a}\left(\theta_{0}\right)$. This allows us to write

$$
\begin{aligned}
\sigma_{\mathrm{II}}= & \frac{1}{\sin \theta}\left\{P_{r}\left[\alpha_{r}-\sigma_{\mathrm{I}}^{r}\left(\theta_{0}\right) \cos \theta\right]+P_{s}\left[\alpha_{s}-\sigma_{\mathrm{I}}^{s}\left(\theta_{0}\right) \cos \theta\right]\right. \\
& \left.+P_{o}\left[\alpha_{o}-\sigma_{\mathrm{I}}^{o}\left(\theta_{0}\right) \cos \theta\right]\right\}
\end{aligned}
$$

and we rewrite the yield curve (23) as

$$
\begin{aligned}
& \left\langle\sigma_{\mathrm{I}}^{a}\left(\theta_{0}\right), \min _{\theta}\left\{P_{r} \sigma_{\mathrm{II}}^{r}\left[\theta, \sigma_{\mathrm{I}}^{r}\left(\theta_{0}\right)\right]+P_{s} \sigma_{\mathrm{II}}^{s}\left[\theta, \sigma_{\mathrm{I}}^{s}\left(\theta_{0}\right)\right]\right.\right. \\
& \left.\left.+P_{o} \sigma_{\mathrm{II}}^{o}\left[\theta, \sigma_{\mathrm{I}}^{o}\left(\theta_{0}\right)\right]\right\}\right\rangle .
\end{aligned}
$$

As $\sigma_{\mathrm{II}}^{r}\left[\theta, \sigma_{\mathrm{I}}^{r}\left(\theta_{0}\right)\right], \sigma_{\mathrm{II}}^{s}\left[\theta, \sigma_{\mathrm{I}}^{s}\left(\theta_{0}\right)\right]$ and $\sigma_{\mathrm{II}}^{o}\left[\theta, \sigma_{\mathrm{I}}^{o}\left(\theta_{0}\right)\right]$ are minimized by $\theta=\theta_{0}$, the yield curve for the full solution of (23) and (24) is given by the locus of points

$$
\left\{\sigma_{\mathrm{I}}^{a}, \min _{\theta} \sigma_{\mathrm{II}}^{a}\left(\sigma_{\mathrm{I}}^{a}, \theta\right)\right\}
$$

Since both $\boldsymbol{\sigma}$ and $\boldsymbol{\sigma}^{a}$ satisfy the plastic work equation (24) and the principle of shear stress minimization (23), it follows that $\boldsymbol{\sigma}=\boldsymbol{\sigma}^{a}$.

Since $\alpha_{o}=\alpha_{r}+\cos \theta$, we can write (27) as

$$
\left(\sigma_{\mathrm{I}}^{o}-1\right) \cos \theta+\sigma_{\mathrm{II}}^{o} \sin \theta=\alpha_{r}(\theta),
$$

so, at a point on the yield curve, $\sigma_{\mathrm{I}}^{o}=1+\sigma_{\mathrm{I}}^{r}, \sigma_{\mathrm{II}}^{o}=\sigma_{\mathrm{II}}^{r}$,

$$
\boldsymbol{\sigma}^{o}(\dot{\boldsymbol{\epsilon}})=\mathbf{1}+\boldsymbol{\sigma}^{r}(\dot{\boldsymbol{\epsilon}})
$$

and the normalized yield curve for opening is determined by that for ridging. This means that the general sea ice stress expression (28) can be written in terms of the ridging and sliding stress contributions only,

$$
\boldsymbol{\sigma}(\dot{\boldsymbol{\epsilon}})=P_{o} \mathbf{1}+\left(P_{r}+P_{o}\right) \boldsymbol{\sigma}^{r}(\dot{\boldsymbol{\epsilon}})+P_{s} \boldsymbol{\sigma}^{s}(\dot{\boldsymbol{\epsilon}})
$$

\section{Ice thickness dependence of ridging and sliding strengths}

Up to this point, we have not considered the particular dependence of the ice strengths in ridging and sliding of a uniform ice layer upon the ice thickness. Several functional forms for the dependence of ridging ice strength on ice thickness have been used in the past. Rothrock (1975) related ice strength in convergence to the change in potential energy involved in forming a pressure ridge (and keel). This led Overland and Pease (1988), Holland (2001), and Wilchinsky and Feltham (2004) in their single-ice-layer models to set the ridging ice strength proportional to $h^{2}$. This approach produces the multilayer ice strength in the form

$$
P_{r}^{R}=C_{f} C_{p} \int_{0}^{\infty} \psi h^{2} d h, C_{p}=1 / 2\left(\rho_{i} / \rho_{w}\right) b\left(\rho_{w}-\rho_{i}\right),
$$

where $b$ is gravitational acceleration, and $C_{f}$ accounts for the frictional ridging (Hopkins 1994) and is usually taken to be 17 (Flato and Hibler 1995). Another sea ice strength formula in current use for both two-layer and multilayer sea ice models is a linear relation between ridging ice strength and the mean thickness proposed by Hibler (1979),

$$
P_{r}^{H}=P^{*} H e^{-C_{H}(1-C)},
$$

where $H$ is the mean ice thickness, $C$ is the relative area covered in sea ice, and $P^{*}$ and $C_{H}$ are parameters, with typical values of $P^{*}=5000-27500 \mathrm{~N} \mathrm{~m}^{-1}$ and $C_{H}=20$.

When only one crack in sea ice is considered, then the ridging and sliding forces determine the corresponding ice strengths. Discrete element simulations by Hopkins (1998) carried out for a particular set of material parameters reveal that a pressure ridge forms by growth of the sail until a buckling threshold is reached. The force necessary to increase sail height is determined by pushing a train of blocks over the sail surface, which, for the adopted values of material parameters, is equal to $7300 h^{3 / 2} L^{1 / 2} \mathrm{~N} \mathrm{~m}^{-1}$, where $L$ is the length of lead ice pushed into the ridge. The next phase starts when this force reaches the buckling force, $95400 h^{3 / 2} \mathrm{~N}$ $\mathrm{m}^{-1}$, which happens always at the same $L=L_{f}=107.7$ $\mathrm{m}$. Although in the context of a continuum sea ice dynamics model, it is difficult to determine which stage of ridge building is occurring; it is noteworthy that both ridging forces are proportional to $h^{3 / 2}$.

The choice of a specific transfer function $\beta\left(h, h^{\prime}\right)$ adopted in a continuum sea ice model may not necessarily correspond to the transfer function recovered from a discrete element simulation and so we now con- 
sider how a particular choice of the sea ice transfer function may affect the ice strength. We consider the transfer function $\beta\left(h, h^{\prime}\right)$ introduced by Hibler (1980) that corresponds to the buildup of a ridge of triangular shape: uniform ice is redistributed between 2 times the thickness of the ice being ridged and a maximum ridge thickness of $2 \sqrt{H^{*} h}$,

$$
\beta\left(h, h^{\prime}\right)=\frac{1}{2\left(H^{*}-h\right)}, \quad 2 h \leq h^{\prime} \leq 2 \sqrt{H^{*} h} .
$$

From this, a ridge formed from a uniform sea ice layer of thickness $h$ has a fractional area of $\left(1+\sqrt{H^{*} / h}\right)^{-1}$. For ice of thickness $h=1$ the fractional ridge area is estimated as 0.1 when $H^{*}=100$ (Hibler 1980) and 0.2 when $H^{*}=25$ (the standard value in the Los Alamos CICE code). In his analysis, Hibler assumed the aspect ratio of ridges to be constant, which means that the angle between the ridge surface and the vertical, $\phi$, is also constant. In this case, since a keel is typically 5.5 times the sail (Sisodiya and Vaudery 1981), the sail height is about $2 \sqrt{H^{*} h} / 6.5$. The component of the force of gravity perpendicular to the sail surface per unit length of the sail, that is, the normal traction, is given by $\rho h b \sin \phi$, where $b$ is the acceleration due to gravity. This determines the frictional force acting on the train of blocks, of length $L$, that are pushed into the ridge as $\mu \rho h b \sin \phi L$, where $\mu$ is the coefficient of ice friction. Since the sail surface length is about $2 \sqrt{H^{*} h} /$ $(6.5 \cos \phi)$, the work done by moving the train of ice blocks over the sail surface is estimated (as not all blocks move along the whole surface) as (2/6.5) $L \mu \rho b$ $\tan \phi h^{3 / 2} b \sqrt{H^{*}}$. Since the ridge area is small in comparison with the ice sheet consumed in its formation, the fraction of the ridged ice is effectively unity, which, in turn, determines a unit ridging deformation. Therefore the work per unit ridging deformation, which is the ridging ice strength, is given by

$$
P_{r}^{*} \lesssim 0.3 \mu \rho b \tan \phi \sqrt{H^{*}} h^{3 / 2} .
$$

(The inequality arises because we considered the maximum ridge height, whereas not all ridges will reach this height and so the ridging work would be less.) If we assume that the ice friction coefficient $\mu$, accounting for both dry and wet friction, does not depend on the ridge form, then the ridging ice strength in similar triangular ridges is affected only by the parameters $\phi$ and $H^{*}$. In particular, for the same ridge aspect ratio $(\phi=$ const $)$, the ridging ice strength is proportional to $\sqrt{H^{*}}$. When all parameters in (39) are fixed, we can unite the constants not altered in the model of redistribution into one to write

$$
P_{r}^{*}=\xi h^{3 / 2},
$$

where the constant of proportionality $\xi$ cannot exceed a value determined by the ice buckling force, which for a particular set of material parameters was found by Hopkins (1998) to be $95400 \mathrm{~N} \mathrm{~m}^{-1}$. Thus, the ridging ice strength calculated using the transfer function (39) is proportional to $h^{3 / 2}$ in agreement with the discrete element approach.

We now consider the sliding strength. If sliding along a floe edge was always accompanied by ridging along the edge, then the sliding force could be related to the ridging force by a frictional law, $P_{s}^{*}=\mu P_{r}^{*}$, where $\mu$ is the coefficient of friction of ice, which we will assume to be constant. In reality, sliding need not be accompanied by ridging so that typically the normal stress exerted across a sliding floe edge can be less than that determined by the ridging force.

This may be modeled by adopting the relationship

$$
P_{s}^{*}=k P_{r}^{*}
$$

where the value of the constant $k$ differs from that of $\mu$ in order to account for lower sliding friction and which can be determined by comparing model results with observations. Use of the parameter $k$ (rather than $\mu$ ) enables the dynamical characteristics of floe motion, not captured by the simple kinematic model of UM, to be partly accounted for. In particular, as can be seen from (22), if $P_{s}^{*}=\mu P_{r}^{*}$ is used, then a decrease of the ice friction coefficient would lead to a decrease in the ratio of sliding power to ridging power whereas dynamic, discrete element modeling by Hopkins (1996) revealed the exact opposite: a decrease of the coefficient of friction implies that less energy is required to slide floes than to ridge them in some places, which leads to a higher amount of sliding and, consequently, a higher deformation power due to sliding. Despite such a difference in treating the sea ice deformation power as a function of the coefficient of friction, the partition into ridging and sliding power given by the simple kinematic model of UM and (42) reproduces the partition calculated with the dynamic model of Hopkins (1996) reasonably accurately, as can be seen in Fig. 3. From (41) and (42), we assert that the sliding ice strength of a uniform layer is given by

$$
P_{s}^{*}=k \xi h^{3 / 2} .
$$

No clear understanding, and therefore no common agreement, have yet been attained by sea ice researchers as to the role of the opening strength (tensile strength) in sea ice models. Laboratory-scale experiments show that the tensile strength is about $1 / 10$ of the compressive strength (Schulson and Nickolayev 1995). 


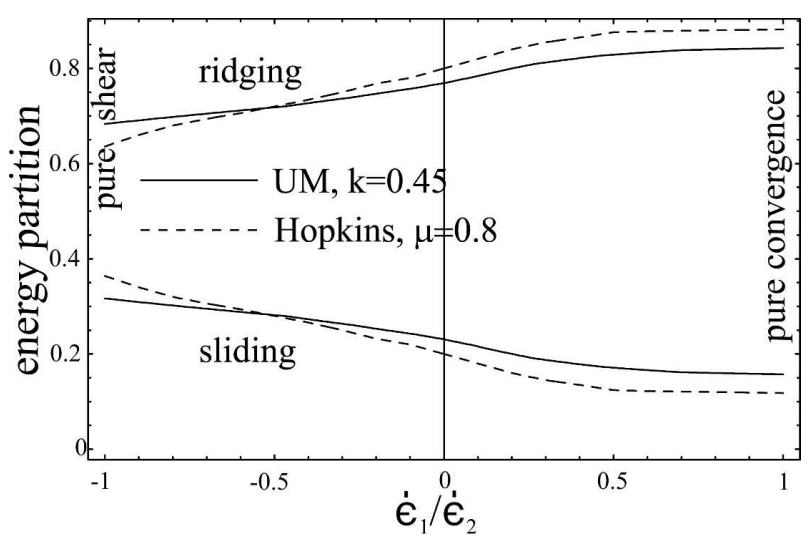

FIG. 3. The fraction of the rate of work going into ridging and sliding using the UM kinematic model with $k=0.45$ and the dynamic model of Hopkins (1996) with $\mu=0.8$.

On the other hand, in situ measurements during the Sea Ice Mechanics Initiative (SIMI) experiment, for example, Lewis and Richter-Menge (1998), recorded tensile stresses of around $75 \mathrm{kPa}$, whereas compressive stresses were around $25 \mathrm{kPa}$. The same typical compressive stresses were recorded during the Surface Heat Budget of the Arctic Ocean (SHEBA) field experiment (Richter-Menge et al. 2002). Even though the tensile strength is small, it may play a role in areas of low tensile wind force. A nonzero tensile strength is known to cause instabilities (Gray 1999) that can be both numerical and mechanical. Here we aim at studying how the adopted yield curve shape, ridging strength, and ridging rate will affect the sea ice evolution. The importance of tensile strength would require a separate study, and thus in the following analysis, for the purposes of clarity, we neglect any contribution to ice strength from opening deformation, that is, $P_{o}^{*}=0$.

Let us consider the yield-curve shape sensitivity to variation in $k$. Adopting (43) for the sliding frictional ice strength means the ratio of the ridging force at two different ice thicknesses is equal to the similar ratio of the sliding force, $P_{r}^{*}\left(h_{1}\right) / P_{r}^{*}\left(h_{2}\right)=P_{s}^{*}\left(h_{1}\right) / P_{s}^{*}\left(h_{2}\right)$. Therefore, we assume that the change of the fractions of the sea ice involved in ridging and sliding depends upon ice thickness in the same way so that $\gamma_{r}=\gamma_{s}$ and $a_{r}=a_{s}$. In this case the relative contribution from sliding and ridging to the rate of doing plastic work is given by

$$
\frac{P_{s}}{P_{r}}=k\left[1-\int_{0}^{\infty} \int_{0}^{\infty} \beta\left(h^{\prime}, h^{\prime \prime}\right) a_{r}\left(h^{\prime}\right) d h^{\prime} d h^{\prime \prime}\right]
$$

and, for a fixed $k$, this ratio depends only upon the ice thickness distribution. Equation (44) identifies the de-

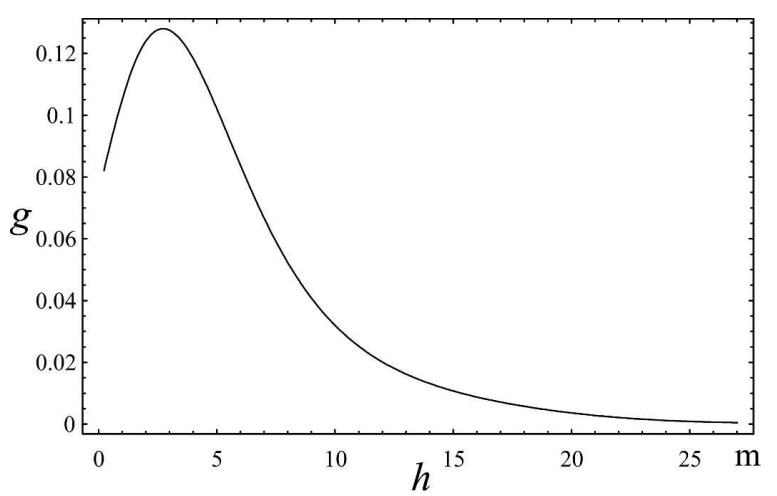

FIG. 4. Mean ice thickness distribution within regions A and B of the Arctic as described by Wadhams (1987) in May 1987. Zone A extends from the North Pole to $85^{\circ} \mathrm{N}$, and Zone B extends from $85^{\circ}$ to $82^{\circ} \mathrm{N}$ across the north of Greenland.

gree to which the sea ice yield-curve shape varies when the ice thickness distribution changes. The variability of this ratio is determined by the term $\int_{0}^{\infty} \int_{0}^{\infty} \beta\left(h^{\prime}\right.$, h) $a_{r}\left(h^{\prime}\right) d h^{\prime} d h$, which is the area of the ridges formed by ridging of a unit area of ice. The latter was estimated to be 0.1 for $H^{*}=100$ for the transfer function (39), so the ice strength ratio variability is estimated as $0.1 k$, which, given $k \leq 1$, is not greatly significant.

Here, we present an example yield curve calculation using (33), (41), and (43) for a particular ice thickness distribution. We considered a smooth approximation of an ice thickness distribution inferred from submarine measurements of draft in May 1987 within two regions: Zone A extends from the North Pole to $85^{\circ} \mathrm{N}$ and Zone B extends from $85^{\circ}$ to $82^{\circ} \mathrm{N}$ across the north of Greenland (Fig. 6 in Wadhams 1987). The ice thickness distribution in Zone A was derived from a $600-\mathrm{km}$ profile. No data are available on the length of profile in Zone B.

The draft-to-thickness conversion factor was taken as 1.126 (Comiso et al. 1991) and the smoothed thickness distribution function $g(h)$ appropriate for both these regions is shown in Fig. 4. The normalized yield curve (36) with the normalized ridging and sliding contributions described in the appendix and ice strengths (41) and (43) are shown in Fig. 5 with $k$ set to 0.2, 0.6, and 1. Also shown in Fig. 5 is the elliptic yield curve of Hibler (1979) with aspect ratio equal to $1 / 2$. For $k=0.6$ and 1 , the shear strength of our yield curve is greater than two times larger than that of the elliptic curve.

A number of yield curve shapes have been proposed by sea ice modelers (see Fig. 6). The most widely used is an elliptic one (Hibler 1979). As an aggregation of ice floes resembles a granular material, Tremblay and Mysak (1997) used a Mohr-Coulomb-type triangular yield curve. A sine-wave yield curve was obtained by Bratchie (1984) studying inelastic floe collision. An ice- 


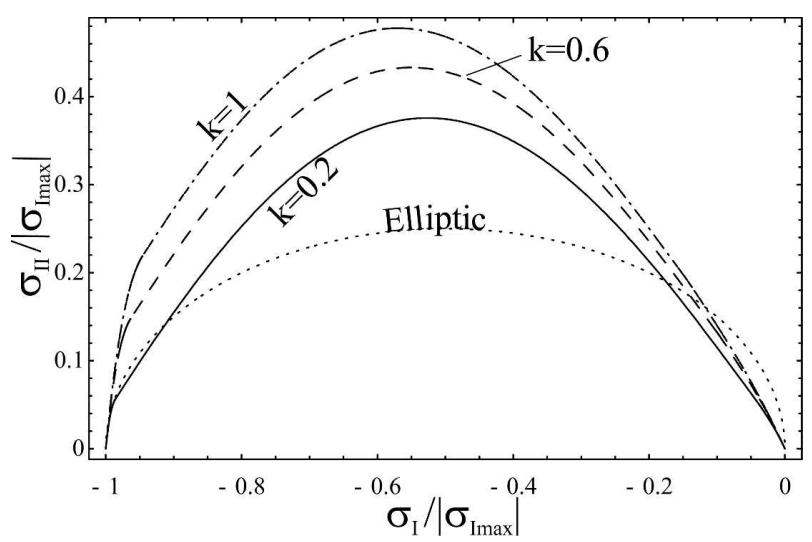

FIG. 5. The normalized yield curves found using the ice thickness distribution within regions $\mathrm{A}$ and $\mathrm{B}$ for $k=0.2$ (solid), $k=$ 0.6 (dashed), and $k=1$ (dotted-dashed), as well as the elliptic yield curve with an aspect ratio equal to $1 / 2$ (dotted).

cream-cone yield curve was originally adopted by Coon (1972), who considered the analogy of sea ice with a granular soil. Laboratory experiments produced similar results (Schulson and Nickolayev 1995) that have been used for larger-scale modeling, for example, Hibler and Schulson (2000). Yield curves of a similar droplet shape have been produced by considering floe collision (Shen et al. 1987), applying the method of characteristics (Pritchard 1988), discrete element simulation (Hopkins 1996), and studying the effect of the presence of two leads (Hibler and Schulson 1997). Although ice-creamcone and Mohr-Coulomb-type yield curves are used in large-scale Arctic simulations (Armstrong et al. 2003; Heil and Hibler 2002), the most commonly used yield curve still remains an elliptic yield curve. Depending on a particular value of the parameter $k$, the yield curve shape used here can change from that determined by only ridging $(k \rightarrow 0)$, which is more similar to a sinewave shape, to that determined by sliding $(k \rightarrow \infty)$, which has a shape of an imperfect ice cream cone. The sea ice dynamics are determined not only by the yield curve shape but also the flow rule.

\section{Simulations of the Arctic sea ice cover}

We have incorporated our new model of sea ice rheology into the Los Alamos CICE sea ice model code (version 3.0, freely available online at http:// climate.lanl.gov/Models/CICE/), which describes both the thermodynamic and dynamic evolution of the sea ice cover. A full description of the Los Alamos CICE sea ice model code is contained in the user manual (Hunke and Lipscomb 2001). To incorporate our new sea ice rheology, we changed the sea ice strengths for

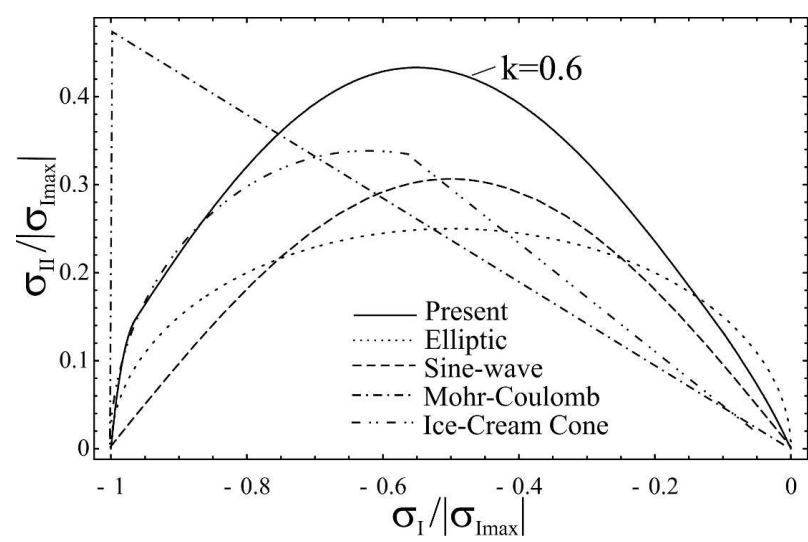

FIG. 6. Some yield curve shapes used in sea ice modeling. The proposed yield curve is plotted for $k=1$.

ridging and sliding, now given by (43); changed the area loss rate due to ridging, which is now given by $|\dot{\epsilon}| \alpha_{r}(\theta)$; and inserted a new routine for calculating sea ice stress. The sea ice stress calculation was modified to include our sea ice rheology, but following Hunke and Dukowicz (1997) we introduced artificial elasticity. Elasticity was included not to describe any physical effect, but to make use of an efficient, explicit numerical algorithm used to solve the full sea ice momentum balance. We represent the plastic sea ice rheology in standard, reduced Reiner-Rivlin form:

$$
\boldsymbol{\sigma}=\nu(\dot{\boldsymbol{\epsilon}}) \mathbf{1}+2 \eta(\dot{\boldsymbol{\epsilon}}) \dot{\boldsymbol{\epsilon}}^{*},
$$

where $\dot{\boldsymbol{\epsilon}}^{*}$ is the traceless part of the strain rate and plasticity is ensured by homogeneity of the scalar coefficients $\nu$ and $\eta$ with respect to strain rate magnitude of order zero and minus unity, respectively. The scalar coefficients, interpreted as viscosities, are given by

$$
\nu=\sigma_{\mathrm{I}}[\theta(\dot{\boldsymbol{\epsilon}})], \eta=\sigma_{\mathrm{II}}[\theta(\dot{\boldsymbol{\epsilon}})] / \dot{\epsilon}_{\mathrm{II}},
$$

where $\sigma_{\mathrm{I}}(\theta), \sigma_{\mathrm{II}}\left(\sigma_{\mathrm{I}}\right)$ are given by (29) and (30) and $\sigma_{I}^{r, s}(\theta), \sigma_{I I}^{r, s}\left(\sigma_{I}^{r, s}\right)$ are represented through the polynomial approximations given in the appendix. The elasticviscous-plastic rheology is given by

$$
T \frac{\partial \boldsymbol{\sigma}}{\partial t}+\boldsymbol{\sigma}=\nu(\dot{\boldsymbol{\epsilon}}) \mathbf{1}+2 \eta(\dot{\boldsymbol{\epsilon}}) \dot{\boldsymbol{\epsilon}}^{*},
$$

where $T$ is an elastic relaxation time scale and we have written a partial time derivative instead of an objective corotational time derivative because the advectional terms are relatively small (Hunke and Dukowicz 1997). In the case of an elliptic yield curve with aspect ratio $1 / e_{a}$, the above model becomes identical with the elastic-viscous-plastic (EVP) model of Hunke and Dukowitz (1997) if the elastic relaxation time for the isotropic part of the stress $\sigma_{\mathrm{I}}$ is taken to be $e_{a}^{2}$ times that for 
tension and shear stresses. An explicit time-stepping algorithm was implemented into the Los-Alamos CICE sea ice code

$$
T \frac{\hat{\boldsymbol{\sigma}}-\boldsymbol{\sigma}}{\Delta t_{e}}+\hat{\boldsymbol{\sigma}}=\nu(\dot{\boldsymbol{\epsilon}}) \mathbf{1}+2 \eta(\dot{\boldsymbol{\epsilon}}) \dot{\boldsymbol{\epsilon}}^{*},
$$

where $\Delta t_{e}$ is the elastic subcycling time step and the stress value at the end of the time step is identified by a caret. For zero strain rate the viscosity coefficients are not determined. In this case, we assume that, when the deformation has stopped, the stress remains the same.

The modified Los Alamos CICE sea ice model was run in stand-alone mode with external forcing data identical to that adopted by Miller et al. (2005). In particular, 40-yr European Centre for Medium-Range Weather Forecasts (ECMWF) Reanalysis (ERA-40) data were used to impose 6-hourly 10-m winds, snowfall, longwave and shortwave radiation, and daily 2-m temperatures were adopted from the Polar Exchange at the Sea Surface (POLES)/International Arctic Buoy Programme (IABP) data. The oceanic heat flux was calculated from a mixed layer model, while the iceocean drag was found from the temporally constant, but spatially varying, ocean currents of Zhang et al. (1998). The model was run on a rotated latitudelongitude grid with $1^{\circ}$ resolution and was spun up for 10 years using 1980 forcing repeatedly, after which it simulated Arctic sea ice from 1980 to 2001. The sea ice thickness distribution function was represented through the open water fraction and nine ice thickness categories of varying size with higher resolution for thinner ice. The modified routine for calculating sea ice stress showed slightly less accuracy than the standard elasticviscous-plastic rheology with an elliptic yield curve and it was necessary to choose a subcycle time step $\Delta t_{e}$ of 36 $\mathrm{s}$ instead of the standard $90 \mathrm{~s}$.

\section{a. Comparison between observed and modeled ice thickness}

Several model runs were performed and the predictions of sea ice thickness were compared with two datasets: European Remote Sensing Satellite (ERS) altimeter-derived ice thickness for the months of November to April between 1993 and 2001 below $81.5^{\circ} \mathrm{N}$ (Laxon et al. 2003; Miller et al. 2006) and the ice draft derived from digitally recorded upward-looking sonar draft data from nine U.S. and U.K. submarine cruises between 1987 and 1997, described in Rothrock et al. (2003). The areas covered by ERS and the submarine cruises are shown in Figs. 7a and 7b. The submarine cruise drafts are given in terms of their mean values averaged over linear segments of 10-50 km.

The model simulations differed in two main respects: (i) sea ice rheology, with either the EVP model with elliptic yield curve with aspect ratio of $1 / 2$ (denoted "Hibler") or the rheology presented in this paper (denoted "Present"); (ii) ice strength, for which three parameterizations of ridging strength were tested: the strength law of Rothrock (37) (denoted "Rothrock"), the strength law of Hibler (denoted "Hibler"), and the strength law presented in this paper (16), (18), and (43) (denoted "Present"). When the Hibler or Rothrock ridging ice strengths were used with the Present rheology, the sliding ice strength was determined from (44). The Present ice strength was not used together with an elliptic yield curve since the nonmodified CICE code was used for the Hibler (elliptic) yield curve. The ridging ice strength constants $\xi, P^{*}$, and $C_{f}$ given in Table 1 were set to provide the best match with the ERS-derived ice thickness data in winter 2001/02, and the fraction of sliding to ridging strength $k$ was set to 0.6 except where otherwise stated. The rheology and ice strength combinations are identified by pairs in parentheses, where the first term identifies rheology and the second term identifies ridging strength; for example, (Present, Hibler) means that the rheology described here was used together with the Hibler ice strength formula.

In terms of the evolution of the mean ice thickness over the area covered by the ERS data, all models performed similarly with only slight differences in different years, Fig. 8. Usually models employing the Hibler ice strength produced lower mean ice thickness in 1996-99. The differences between model configurations is more pronounced when the spatial distribution of the mean ice thickness over the same area is compared, Fig. 9. Here, it is seen that the combination of rheology and ice strength presented in this paper (Present, Present) performed better than the other configurations. In particular, there is much greater difference between the measured and modeled ice thickness for ice thicknesses smaller than $2 \mathrm{~m}$ when the Hibler rheology is used, or the Present rheology with the Hibler or Rothrock ice strengths. When the Present rheology is used then the

FIG. 7. Spatial plots for the (first column) time-average observed data, (second column) (Present, Hibler) model bias, and (third column) (Hibler, Hibler) model bias. (a) The ERS ice thickness data, (b) submarine-derived ice drafts, and (c) and (d) Polar Pathfinder ice speeds and velocity angles (with respect to $90^{\circ}$ lon). 
(a)

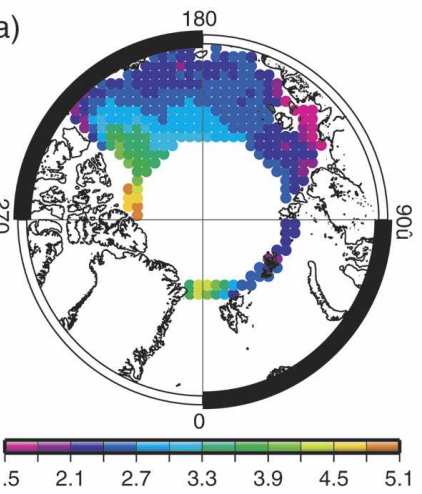

(b)
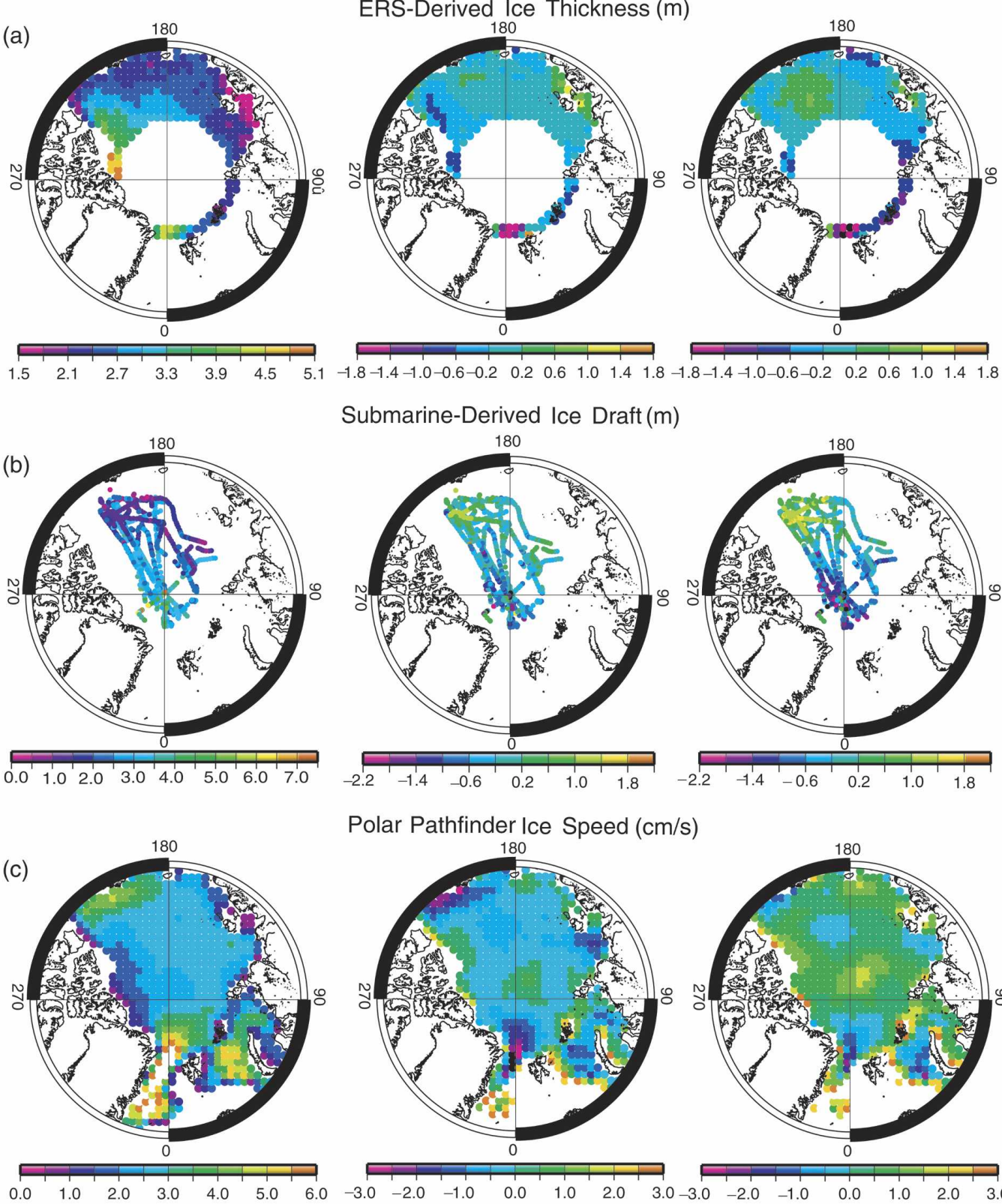

Submarine-Derived Ice Draft (m)
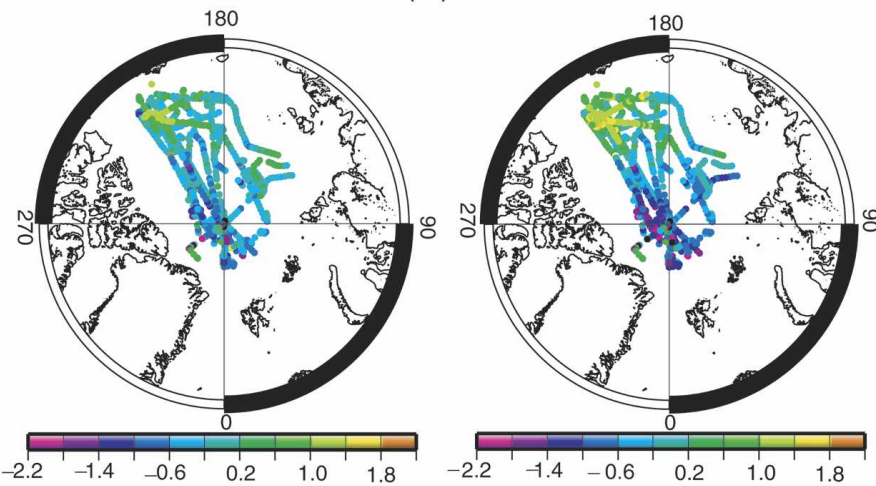

Polar Pathfinder Ice Speed $(\mathrm{cm} / \mathrm{s})$
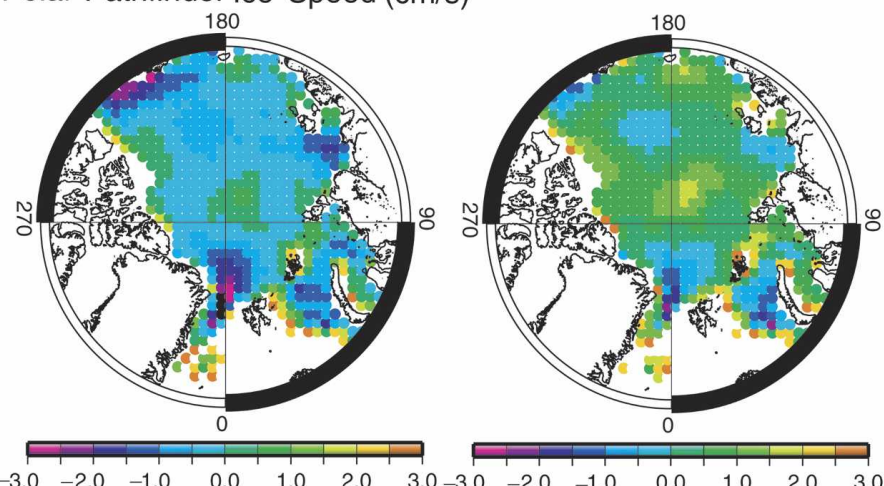

Polar Pathfinder Ice Velocity Angle (deg.)
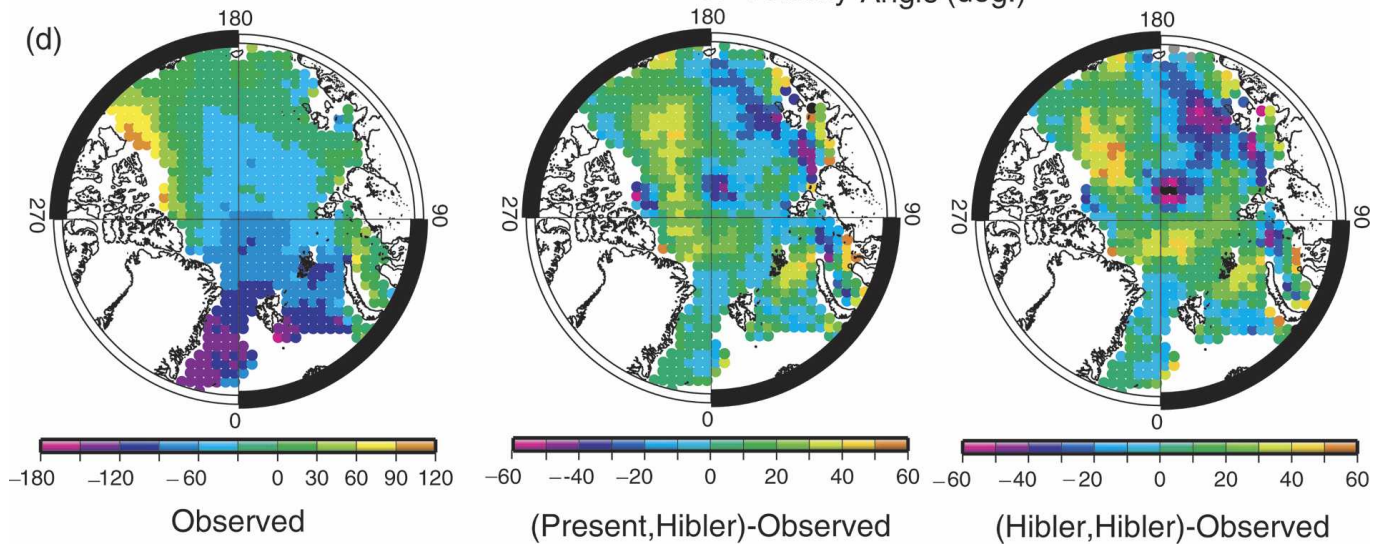

(Present,Hibler)-Observed

(Hibler,Hibler)-Observed 
TABLE 1 . Model parameters $\xi, P^{*}$, and $C_{f}$ chosen to optimize the model fit with ERS data as $k$ changes. Pr.ls.shear is Present less shear.

\begin{tabular}{llcccc}
\hline \hline Rheology & $\begin{array}{c}\text { Ice } \\
\text { strength }\end{array}$ & $k$ & $\begin{array}{c}\xi / 95400 \\
\left(\mathrm{~N} \mathrm{~m}^{-1}\right)\end{array}$ & $\begin{array}{c}C_{f} \\
\left(\mathrm{kN} \mathrm{m}^{-1}\right)\end{array}$ \\
\hline Present & Present & 0.2 & 0.5 & - & - \\
Present & Present & 0.6 & 0.4 & - & - \\
Present & Present & 1 & 0.327 & - & - \\
Present & Rothrock & 0.6 & - & 7.2 & - \\
Present & Hibler & 0.2 & - & - & 14 \\
Present & Hibler & 0.6 & - & - & 11.5 \\
Present & Hibler & 1 & - & - & 9.7 \\
Pr.ls.shear & Hibler & 0.2 & - & - & 13.4 \\
Hibler & Hibler & - & - & - & 8 \\
Hibler & Rothrock & - & - & 8.5 & - \\
\hline
\end{tabular}

largest difference at the peaks is produced using the Hibler ice strength, while the smallest difference is produced using the Present ice strength. Computations show that an increase of the ice strength leads to a shift of the peaks to the left, corresponding to a smaller mean ice thickness and vice versa. The peak shape depends on the rheology and ice strength formula used, which is especially evident for the Hibler rheology with the Hibler and Rothrock ice strengths. As the data and model ice thickness over the entire observed domain were used to fit the model parameters in Table 1, the model-data discrepancy around the peak area and the small and large thickness arms are correlated: An underestimation of the ice thickness around the peak is accompanied by an overestimation of the ice thickness in the arms. Furthermore, as the tops consist of several local maxima, the positions of the highest peaks do not coincide.

Scattergrams of the monthly ice thicknesses averaged over the observed area are shown in Fig. 10 with the linear interpolations shown by the dashed lines. Here again the Present model produces a better fit than the others.
The statistics of the model results are presented in Table 2. The correlation, denoted as "Cor," is the spatial correlation: it is calculated by considering the correlation of the monthly ice thickness over all grid points covered by observations at a particular month and then averaging all such monthly correlations with regard to time. The root-mean-square difference (RMSD) is found similarly by calculating a square root of the mean of the squared difference between the observed and modeled monthly data over all grid points covered by observations at a particular month, and then averaging all such monthly RMSD values with regard to time. Bias is given as the mean difference between the model and observation ice thicknesses. From this, more rigorous, comparison we see that the best fit is given by the model using the Present rheology and Hibler ice strength.

To analyze the difference between the spatial thickness distribution produced by different models in more detail, we consider the submarine-measured ice drafts. As was shown by Rothrock et al. (2003), model runs using a viscous plastic sea ice rheology with an elliptic yield curve with aspect ratio of $1 / 2$ poorly described the spatial ice thickness distribution obtained from U.S. and U.K. submarine cruises between 1987 and 1997. To make such a comparison with our model runs we used the submarine-derived ice drafts averaged over $2^{\circ}$ bins of latitude (Miller et al. 2005). The results of the model runs and the observations are presented in Fig. 11. Generally the new, Present, rheology describes the spatial ice draft pattern better, although there is no significant difference between using the ridging strength of Rothrock or that presented in this paper. All results using multithickness ice strengths are characterized by a prominent ice draft increase south of $74^{\circ} \mathrm{N}$. It is notable that all model configurations predicted ice concentrations (averaged over all cruises) of less than $80 \%$ around $75^{\circ} \mathrm{N}$, whereas in all other latitudes it was more
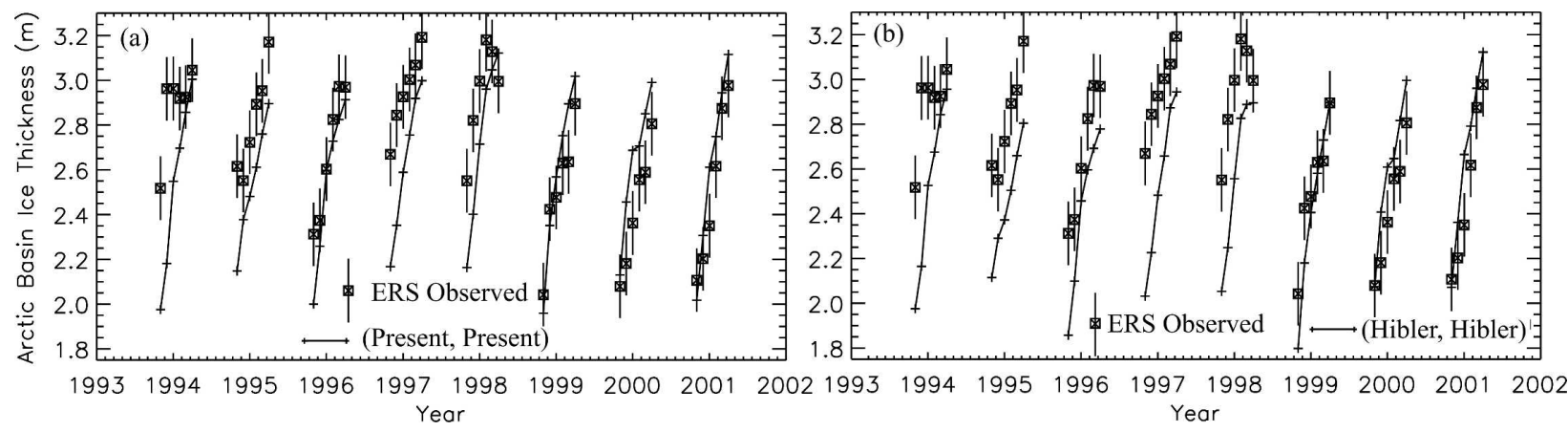

FIG. 8. Temporal evolution of the basin mean ice thickness during winter determined from ERS observations: (a) the Present model and (b) that using the Hibler rheology and ridging strength law in the CICE model. The vertical lines are the error bars. 

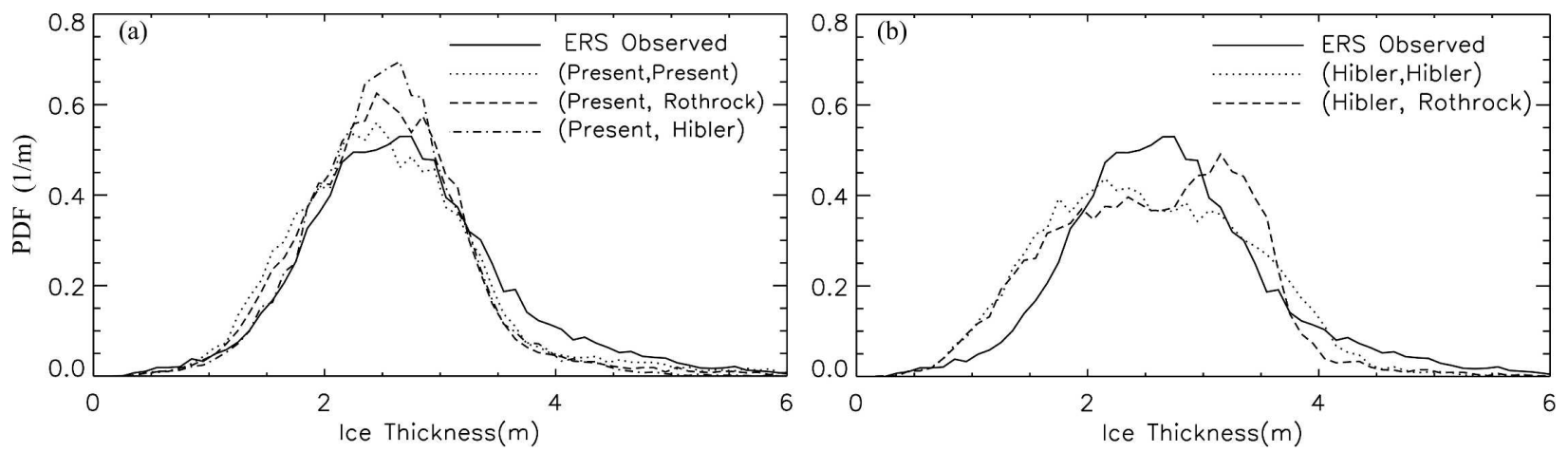

FIG. 9. The PDF of mean ice thickness over the area of thickness observations obtained using ERS given by the (a) Present and (b) Hibler (elliptic) rheological models with different ice strength formulas.

that $90 \%$. As the ice strength depends on the open water fraction, we checked if a change of $C_{1}$ (the fraction of the ice cover undergoing deformation) improved the comparison with observations. It was found that increasing $C_{1}$ from its standard value of 0.15 to 0.3 increased the discrepancy around $73^{\circ} \mathrm{N}$ and decreasing $C_{1}$ to 0.1 made no noticeable difference. Using the rheology presented in this paper with the Hibler representation of ice strength led to the closest match between model predictions and observations.

A sensitivity study was performed to study the influence of the ratio of sliding to ridging strength, that is, the parameter $k$, on the model predictions. As the yield curve shape and size varies as $k$ changes, fitting the ERS data requires choosing different $\xi, P^{*}$, and $C_{f}$ for different values of $k$, presented in Table 1. In Fig. 12, we show a comparison of model predictions of the mean ice draft with the drafts derived from the submarine data reported in Rothrock et al. (2003) for $k$ set equal to $0.2,0.6$, and 1 . The change of $k$ did not produce any significant effect on the spatial distribution of the mean ice draft. From Fig. 2 it is clear that, even if the sliding strength is neglected entirely, $k \rightarrow 0$, the yield curve aspect ratio is still more than 1.3 times that of the standard elliptic yield curve with an aspect ratio of $1 / 2$.

The statistical characteristics for these data are given in Table 2, where all data are given by considering the draft values over all measurements. Again, the model with the proposed rheology and Hibler ice strengths produced the lowest RMSD and the highest correlation.

Miller et al. (2005), who used the CICE sea ice model code (version 3.0), showed that an increase of the shear strength of sea ice can lead to a better spatial ice draft distribution as compared with the submarine data in Rothrock et al. (2003). This was achieved using an elliptic yield curve with a reverse aspect ratio of $e_{a}=$ $\sqrt{0.5}$. Although better agreement with the submarine data was achieved, adopting such a high shear-stress yield curve (e.g., when $e_{a}=\sqrt{0.5}$ ) implies significant tensile stress that is not believed to be appropriate for sea ice. In addition to its direct effect on the momentum balance, the increase of the shear strength using the high-shear-stress elliptical yield curve affects the ice draft distribution through the ridging rate determined from the Flato and Hibler (1995) parameterization
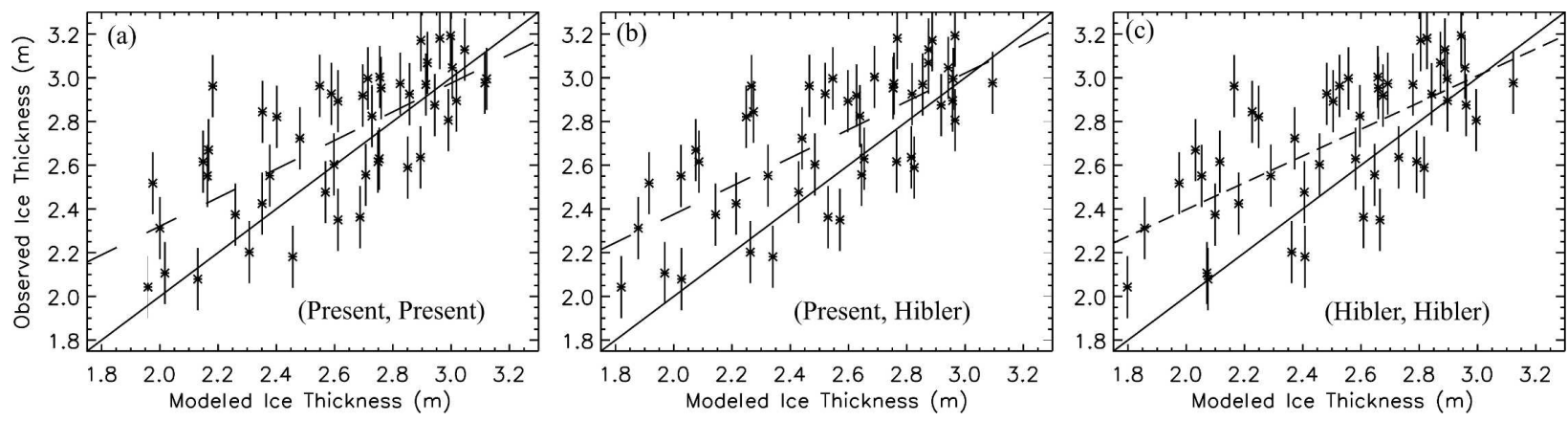

FIG. 10. Scattergrams of the modeled vs ERS-observed monthly mean ice thickness for (a) the Present model, (b) Present rheology with Hibler ice strength, and (c) the (Hibler, Hibler) model. The dashed line shows the linear best fit. The vertical lines are the error bars. 
TABLE 2. Statistical characteristics of the model ice thickness results. The ERS correlation, "Cor," is a spatial correlation over the monthly ice thickness at the grid points averaged over all months between 1993 and 2001. The ERS root-mean-square difference, "RMSD," is a square root of the spatial mean of the squared difference between the observed and modeled monthly data averaged over all considered months. The submarine draft statistics are integrated over all cruise draft points (Fig. 7b). The overall mean ERS-derived ice thickness is $2.72 \mathrm{~m}$. The overall mean submarine-derived ice draft is $2.56 \mathrm{~m}$. RMSD and bias are in meters. Pr.ls.shear is Present less shear.

\begin{tabular}{lllllllrr}
\hline \hline Rheology & Ice strength & $k$ & Cor & RMSD & Bias & Cor & RMSD & Bias \\
\hline Data source & $\rightarrow$ & & ERS & ERS & ERS & Sub & Sub & Sub \\
\hline Present & Present & 0.2 & 0.525 & 0.888 & -0.135 & 0.674 & 0.868 & -0.381 \\
Present & Present & 0.6 & 0.531 & 0.895 & -0.111 & 0.686 & 0.839 & -0.337 \\
Present & Present & 1 & 0.537 & 0.889 & -0.138 & 0.697 & 0.82 & -0.315 \\
Present & Rothrock & 0.6 & 0.518 & 0.802 & -0.181 & 0.709 & 0.821 & -0.352 \\
Present & Hibler & 0.2 & 0.54 & 0.756 & -0.163 & 0.793 & 0.662 & -0.162 \\
Present & Hibler & 0.6 & 0.54 & 0.76 & -0.189 & 0.808 & 0.637 & -0.14 \\
Present & Hibler & 1 & 0.538 & 0.754 & -0.167 & 0.817 & 0.621 & -0.124 \\
Pr.ls.shear & Hibler & 0.2 & 0.526 & 0.778 & -0.182 & 0.74 & 0.739 & -0.213 \\
Hibler & Hibler & - & 0.53 & 0.847 & -0.198 & 0.6 & 0.879 & -0.202 \\
Hibler & Rothrock & - & 0.466 & 0.867 & -0.17 & 0.396 & 1.06 & -0.318 \\
\hline
\end{tabular}

[their (23) with $C_{s} \neq 0$ ]. As the yield curves that we calculate in our model of sea ice rheology, based on the ridging and sliding modes of UM, have a higher shear stress than that given by the elliptic curve of Hibler, this may explain why the Present model produced a better match with the submarine observations. To investigate if it is the higher shear stress or more realistic description of the ridging and sliding modes in our model that leads to better agreement with observations, we performed a model run with $k=0.2$ and the Hibler ice strength but artificially decreased the shear stress of our yield curve by a constant factor to ensure that its aspect ratio is $1 / 2$, identified as "Present less shear" on plots and "Pr.ls.shear" in tables. The results presented in Fig. 13 show that, indeed, although the model performed worse than that with the original nonmodified yield

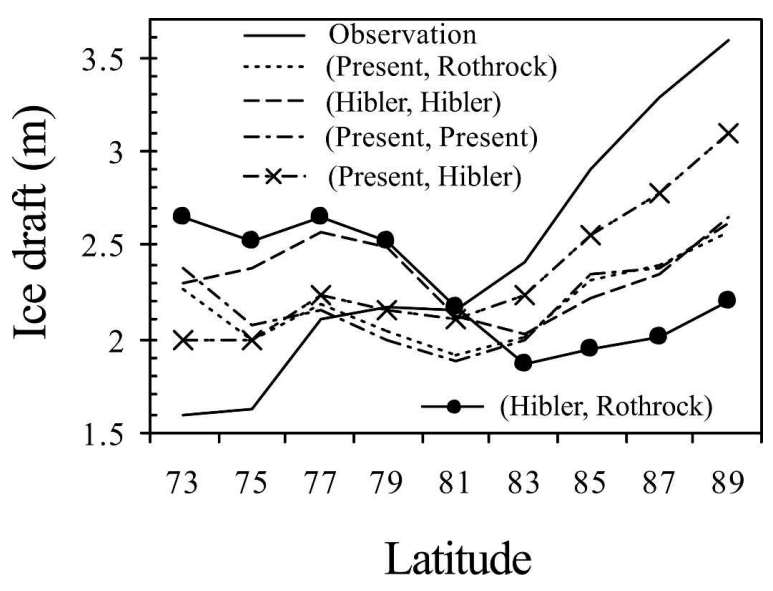

FIG. 11. Ice draft spatial distribution averaged over U.S. and U.K. submarine cruises from 1987 until 1997. The model results are labeled by rheological model and ice strength type. curve, it still produced a better fit with observations than the elliptic curve with the same aspect ratio. This implies that the Present model produces a better match with the submarine observations not only because it ensures a higher shear stress than that of the elliptic yield curve, but also because it describes the ridgingsliding energetics in a more physical way.

\section{b. Comparison between observed and modeled ice velocity}

Gridded, monthly mean ice motion vector fields from the Polar Pathfinder Daily 25-km EASE-Grid Sea Ice Motion Vectors dataset (Fowler 2003) were used to validate the modeled sea ice motion. Daily gridded velocity fields were first created (Fowler 2003) by opti-

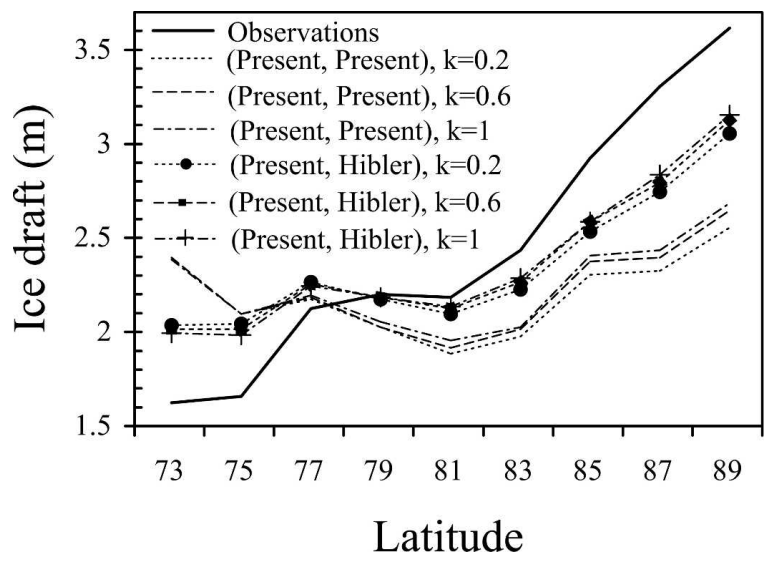

FIG. 12. Sensitivity of model predictions of ice draft to a change in the parameter $k$ and the observations of ice draft obtained by averaging over U.S. and U.K. submarine cruises from 1987 until 1997. 


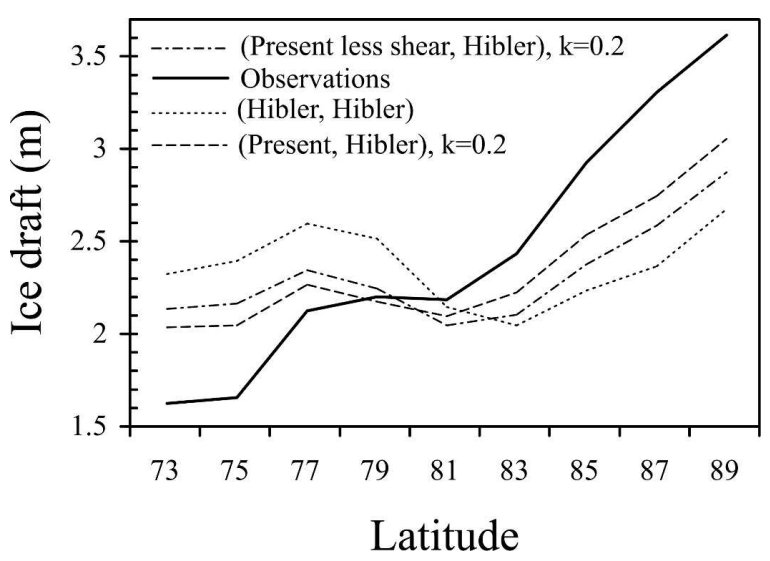

FIG. 13. Sensitivity of model predictions of ice draft to a reduction in shear strength and the observations of the ice draft obtained by averaging over U.S. and U.K. submarine cruises from 1987 until 1997.

mally interpolating data from Scanning Multichannel Microwave Radiometer (SMMR), Special Sensor Microwave Imager (SSM/I), Advanced Very High Resolution Radiometer (AVHRR), and IABP buoy data, and then averaged to produce monthly gridded fields used in this study. We regridded the monthly ice velocity fields between 1994 and 2001 onto the model grid using a Gaussian weighting scheme with a maximum search radius of $100 \mathrm{~km}$. We estimate (Miller et al. 2006) the maximum error in each monthly, grid cell velocity component to be $\Delta_{\text {monthly }} \approx 0.3 \mathrm{~cm} \mathrm{~s}^{-1}$. Basinscale averages over many grid cells further reduce this error.

The area covered by the observation is shown in Figs. $7 \mathrm{c}$ and $7 \mathrm{~d}$, where the data for the ice speed and velocity angle are presented. The velocity angle is calculated from $90^{\circ}$ longitude on the map projected onto a flat surface used in the CICE code. The statistical data for the model runs from 1994 to 2001 are given in Table 3 for all months, Table 4 for three winter months January-March, and Table 5 for three summer months JulySeptember. To calculate the vector correlation (Vec Cor), we used the magnitude of the vector correlation coefficient (Kundu 1976) for two vectors written in the complex space as

$$
\frac{\left\langle w_{1} w_{2}^{*}\right\rangle}{\left(\left\langle w_{1} w_{1}^{*}\right\rangle\left\langle w_{2} w_{2}^{*}\right\rangle\right)^{1 / 2}},
$$

where the angle brackets denote the mean. Also, the speed time correlation (T-Cor) is shown and represents the seasonal correlation between the spatially averaged monthly speed values. Except for the latter, all angle and speed (Spd) statistics were calculated for every month separately and then averaged over time.

The effectiveness of the models in terms of the velocity depends on the measure of success considered. Generally all model vector and seasonal correlations are better for the winter months (Table 4) than for the summer months (Table 5), while the difference between the vector correlation themselves seems not to be significant. If we consider the whole year data (Table 3 ), then the vector correlation is the best for the (Present, Hibler) model, while the seasonal correlation (T-Cor) is best for the (Hibler, Rothrock) model. However, the speed bias is always the best for the (Present, Hibler) model. As the magnitude of speed RMSD compares the model and observed ice speeds at the same grid points, thereby disregarding the difference due to advection, the speed RMSD is much larger than the speed bias, which allows comparison over the whole region. On average the spatial statistics are better for the models using the Present rheology.

The temporal and spatial behavior of the models' velocity is presented in Figs. 14-17. The time evolution of the whole Arctic speed is given in Fig. 14 for (a) 1-month means and (b) 3-month means. The oscillation

TABLE 3. Statistical velocity characteristics of the model results as compared with Polar Pathfinder. Correlation "Vec Cor" is a spatial correlation over the monthly ice velocities at the grid points averaged over all months of 1994-2001. Correlation "T-Cor" is a time correlation over the monthly mean Arctic ice speeds. Speed RMSD is found by considering the spatial mean of the monthly squared speed differences at grid points and averaging with regard to time. The mean absolute angle difference (|AngleDif|) is in degrees; speed (Spd) statistics are in centimeters per second. The overall observed mean ice speed is $2.81 \mathrm{~cm} \mathrm{~s}^{-1}$. Pr.ls.shear is Present less shear.

\begin{tabular}{|c|c|c|c|c|c|c|c|}
\hline Rheology & Ice strength & $k$ & Vec Cor & Spd RMSD & |Angle Dif| & Spd bias & T-Cor \\
\hline Present & Present & 0.2 & 0.767 & 2.0 & 48.7 & -0.184 & 0.603 \\
\hline Present & Present & 0.6 & 0.765 & 2.0 & 48.8 & -0.222 & 0.604 \\
\hline Present & Present & 1 & 0.761 & 2.01 & 49.3 & -0.28 & 0.592 \\
\hline Present & Rothrock & 0.6 & 0.752 & 2.06 & 51.3 & -0.155 & 0.64 \\
\hline Present & Hibler & 0.2 & 0.774 & 1.93 & 48.8 & -0.126 & 0.607 \\
\hline Present & Hibler & 0.6 & 0.773 & 1.93 & 48.8 & -0.17 & 0.601 \\
\hline Present & Hibler & 1 & 0.771 & 1.94 & 48.7 & -0.234 & 0.585 \\
\hline Pr.ls.shear & Hibler & 0.2 & 0.783 & 1.94 & 49.6 & 0.285 & 0.742 \\
\hline Hibler & Hibler & - & 0.781 & 2.02 & 54.3 & 0.603 & 0.81 \\
\hline Hibler & Rothrock & - & 0.787 & 2.06 & 53.0 & 0.668 & 0.834 \\
\hline
\end{tabular}


TABLE 4. Statistical velocity characteristics of the model results as compared with Polar Pathfinder. Correlation "Vec Cor" is a spatial correlation over the monthly ice velocities at the grid points averaged over winter months (Jan-Mar) of 1994 through 2001. Correlation "T-Cor" is a time correlation over the winter monthly mean Arctic ice speeds. The Jan-Mar observed mean ice speed is $2.87 \mathrm{~cm} \mathrm{~s}^{-1}$. Pr.ls.shear is Present less shear.

\begin{tabular}{llllccrc}
\hline \hline Rheology & Ice strength & $k$ & Vec Cor & Spd RMSD & |Angle Dif $\mid$ & Spd bias & T-Cor \\
\hline Present & Present & 0.2 & 0.776 & 2.18 & 55.6 & -0.58 & 0.942 \\
Present & Present & 0.6 & 0.774 & 2.19 & 56.7 & -0.601 & 0.942 \\
Present & Present & 1 & 0.769 & 2.21 & 59.0 & -0.665 & 0.944 \\
Present & Rothrock & 0.6 & 0.767 & 2.24 & 59.2 & -0.573 & 0.945 \\
Present & Hibler & 0.2 & 0.77 & 2.15 & 57.8 & -0.481 & 0.927 \\
Present & Hibler & 0.6 & 0.767 & 2.17 & 57.7 & -0.524 & 0.928 \\
Present & Hibler & 1 & 0.764 & 2.18 & 58.3 & -0.588 & 0.929 \\
Pr.ls.shear & Hibler & 0.2 & 0.79 & 2.11 & 57.3 & 0.055 & 0.899 \\
Hibler & Hibler & - & 0.791 & 2.27 & 61.2 & 0.592 & 0.855 \\
Hibler & Rothrock & - & 0.801 & 2.34 & & 0.67 & 0.872 \\
\hline
\end{tabular}

of 1-month means is quite high, while the 3-month means show that the (Hibler, Hibler) model overestimates the observed ice speed, although its peaks correlate better than those of the (Present, Hibler) model. The probability distribution function (PDF) for the ice speeds for the whole Arctic is shown in Fig. 15 for the (a) Present rheology and (b) Hibler rheology and different ice strength expressions. Again, as in case of the mean ice thickness, the proposed rheology (except when the Rothrock ice strength is used) gives a better fit than the standard elliptic yield curve. The best fit is, however, produced by the (Present, Hibler) variation in contrast to (Present, Present) for the mean Arctic ice thickness PDF (Fig. 9). Similar PDFs for the velocity orientation angle are shown in Fig. 16. The Present rheology either with the Present or Hibler ice strengths produce the best fit with a slightly better match of these two produced by (Present, Present). In all other cases the Angle PDF peak is shifted to the higher negative angles. The shape of the top is the most closely described by the (Present, Hibler) case. In the region of positive angles all models behave similarly. The scattergram for the monthly Arctic ice speeds are given in Fig. 17 for the proposed (Present, Present), (Present, Hibler), and (Hibler, Hibler) models. Although there is an evident bias of the (Hibler, Hibler) model, it is the least scattered around the linear fit, reflecting its high seasonal monthly speed correlation.

\section{Concluding remarks}

We have introduced a new multithickness sea ice model explicitly accounting for the ridging and sliding friction contributions to sea ice stress. Both ridging and sliding contributions depend on the deformation type through functions adopted from the kinematic model of floe interaction of Ukita and Moritz (2000). The model incorporates the difference in the amount of sliding and opening deformation in different ice thicknesses through the introduction of sliding and opening participation functions, in a similar manner to the ridging description of Thorndike et al. (1975).

TABLE 5. Statistical velocity characteristics of the model results as compared with Polar Pathfinder. Correlation "Vec Cor" is a spatial correlation over the monthly ice velocities at the grid points averaged over summer months (Jul-Sep) of 1994 through 2001. Correlation "T-Cor" is a time correlation over the summer monthly mean Arctic ice speeds. Jul-Sep observed mean ice speed is $2.29 \mathrm{~cm} \mathrm{~s}^{-1}$. Pr.ls.shear is Present less shear.

\begin{tabular}{lccccccc}
\hline \hline Rheology & Ice strength & $k$ & Vec Cor & Spd RMSD & |Angle Dif| & Spd bias & T-Cor \\
\hline Present & Present & 0.2 & 0.734 & 1.72 & 56.6 & 0.714 & 0.745 \\
Present & Present & 0.6 & 0.735 & 1.71 & 56.3 & 0.68 & 0.742 \\
Present & Present & 1 & 0.736 & 1.7 & 56.1 & 0.652 & 0.738 \\
Present & Rothrock & 0.6 & 0.732 & 1.73 & 59.2 & 0.705 & 0.753 \\
Present & Hibler & 0.2 & 0.724 & 1.72 & 57.2 & 0.667 & 0.726 \\
Present & Hibler & 0.6 & 0.727 & 1.7 & 56.5 & 0.642 \\
Present & Hibler & 1 & 0.727 & 1.68 & 56.0 & 0.606 & 0.729 \\
Pr.ls.shear & Hibler & 0.2 & 0.72 & 1.81 & 60.1 & 0.724 \\
Hibler & Hibler & - & 0.719 & 1.82 & 60.7 & 0.85 & 0.745 \\
Hibler & Rothrock & - & 0.721 & 1.81 & & 0.857 & 0.755 \\
\hline
\end{tabular}


1-Month Running Mean Arctic Ice Speed

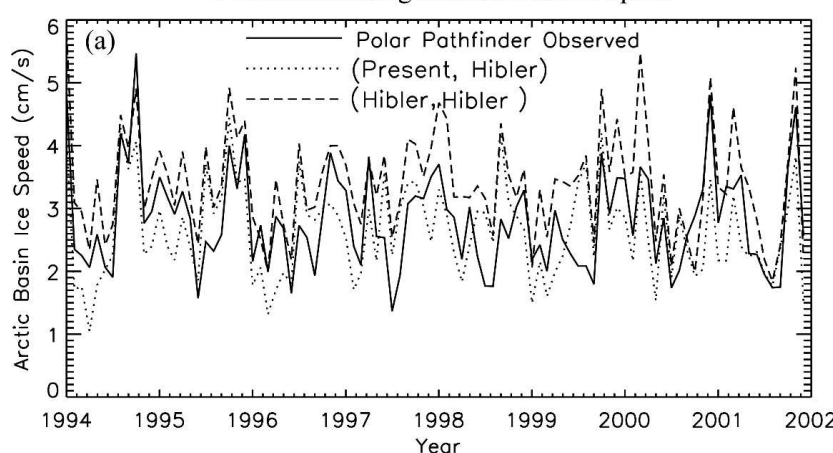

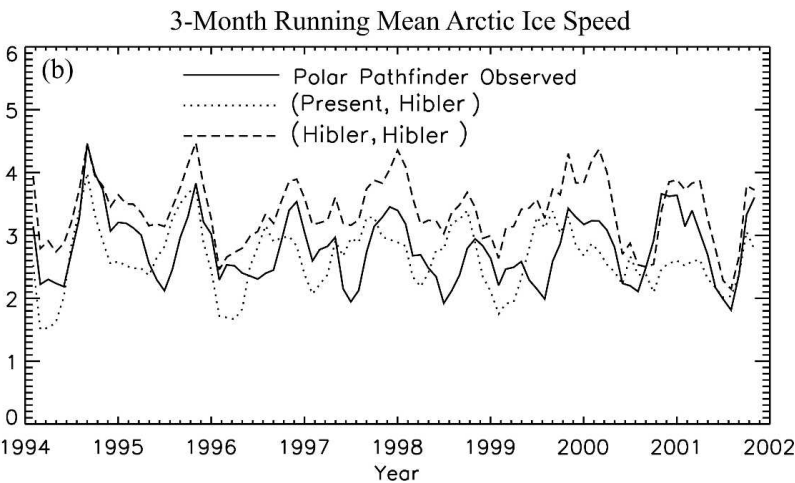

FIG. 14. Temporal evolution of the basin mean ice speed between 1994 and 2001 determined by Polar Pathfinder for the present model with Hibler ice strengths and the (Hibler, Hibler) model: (a) 1-month means and (b) 3-month means.

In contrast to previous work, the ridging ice strength of a uniform ice sheet of constant ice thickness was taken to be proportional to the ice thickness raised to the power of $3 / 2$, as is predicted in the discrete element modeling results of Hopkins (1998). The sliding strength was related to the ridging strength through a linear relationship motivated by a friction law, but the proportionality parameter was treated not as a friction coefficient (which would be true in the case of a purely kinematic approach) but as a parameterization describing the relative ridging and sliding deformation power in a subcontinuum-scale region.

The new multithickness sea ice model for sea ice stress has been implemented into the Los Alamos CICE sea ice model code (version 3.0), which involved generalizing the elastic-viscous-plastic (EVP) rheology module so that it could use a more general plastic rheological law. The results of modeling the sea ice cover over a large region of the Arctic Ocean showed that the proposed model with either the Hopkins or Hibler ice strength performed better when the probability distribution function for the ice thickness, ice speed, and ice velocity angle are considered. However, when comparing model predictions of draft distribution with the submarine observations reported in Rothrock et al. (2003), the proposed rheology with the Hibler ice strengths performed better than all other models including the proposed rheology with the Hopkins ice strengths. Stability problems of the CICE EVP model utilizing the Rothrock ice strengths were reported by Hunke and Lipscomb (2001), which may have contributed to the discrepancy with the submarine measurements but the persistent error in the ice draft distribution produced by all models utilizing multilayer ice strengths suggests this problem is not stability related and will require further analysis. The original Hibler model produced the best result only for the seasonal ice speed correlation.

When the rheology and thickness distribution model presented in this paper is combined with Hibler's ice strength formula, a better agreement between modeled and submarine-measured ice draft distribution as well as ice velocities is achieved than is possible using the standard EVP model. This improvement can be attributed not only to the higher shear stress given by our
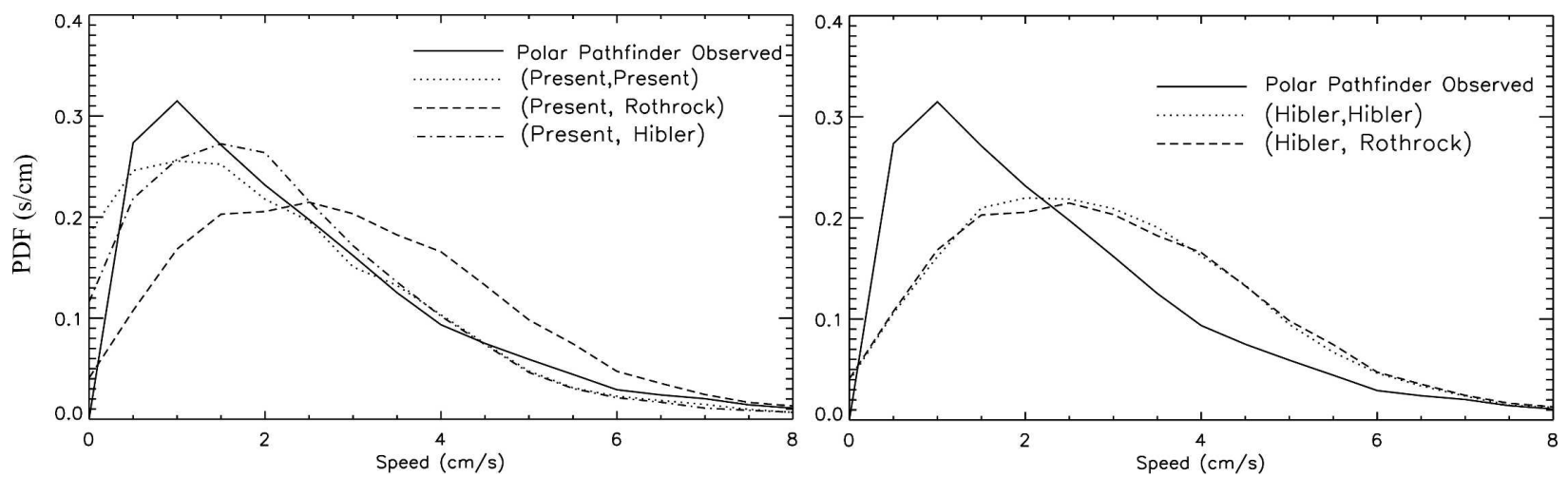

FIG. 15. The spatial PDF of ice speed over the Arctic area averaged between 1994 and 2001 for the (a) Present and (b) Hibler (elliptic) rheological models with different ice strength formulas. 

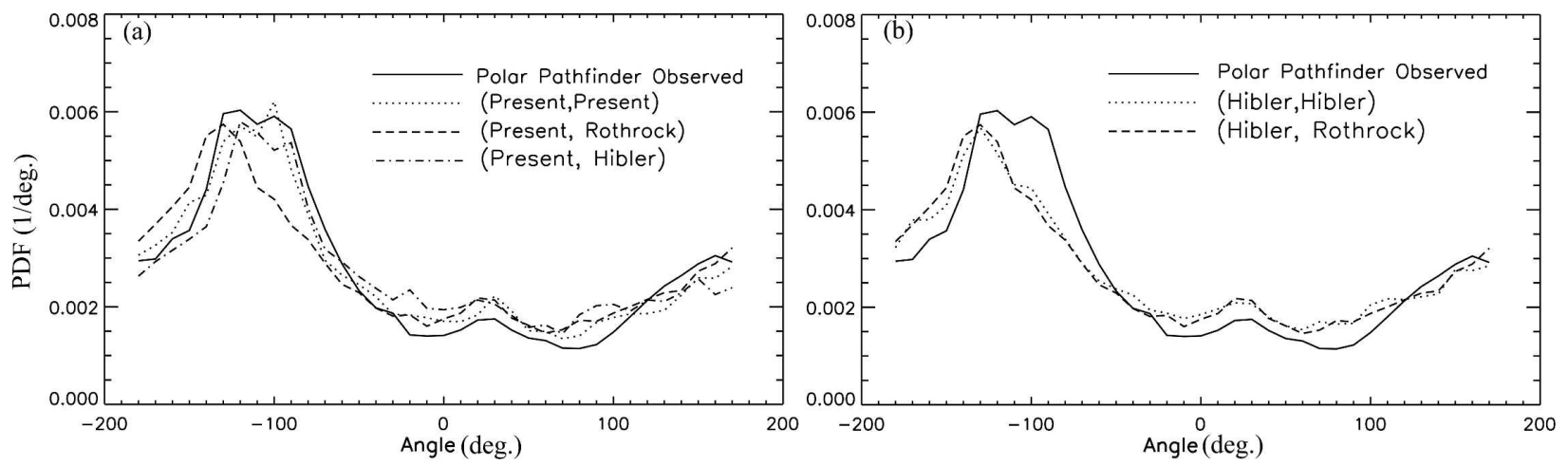

FIG. 16. The spatial PDF of ice velocity vector angle with regard to $90^{\circ}$ lon over the Arctic area averaged between 1994 and 2001 for the (a) Present and (b) Hibler (elliptic) rheological models with different ice strength formulas.

yield curve [which was shown to have a beneficial effect when an elliptic curve was used (Miller et al. 2005)], but also to a more realistic description of the ridging rate recovered from the kinematic model of Ukita and Moritz (2000). Improvements to the spatial distribution of sea ice thickness could be significant for the spatial distribution of surface air temperature and ocean salinity in coupled models since the heat flux through, and growth rate of, the ice in winter is a function of ice thickness. We suggest that the next stage necessary to improve a multithickness sea ice stress and thickness redistribution model would require a more detailed study of the effect of different choices of the ridging and sliding participation functions.

\section{APPENDIX}

\section{Polynomial Expressions for the Normalized Ridging and Sliding Yield Curves}

The solutions $\left\{\sigma_{\mathrm{I}}^{r}(\theta), \sigma_{\mathrm{II}}^{r}\left(\sigma_{\mathrm{I}}^{r}\right)\right\}$ and $\left\{\sigma_{\mathrm{I}}^{s}(\theta), \sigma_{\mathrm{II}}^{s}\left(\sigma_{\mathrm{I}}^{s}\right)\right\}$ to (25) and (26) using the ridging and sliding modes of Ukita and Moritz (2000), presented in Fig. 3, were calculated numerically. Here, we present polynomial approximations that interpolated our calculated results that ensure convexity of the yield curves. We found the following relations $\sigma_{\text {II }}^{r}\left(\sigma_{\mathrm{I}}^{r}\right)$ and $\sigma_{\text {II }}^{s}\left(\sigma_{I}^{s}\right):$

$$
\begin{aligned}
& \sigma_{\mathrm{II}}^{r}=-1.04993 \sigma_{\mathrm{I}}^{r}+0.0517596 \sigma_{\mathrm{I}}^{r 2}+2.04738 \sigma_{\mathrm{I}}^{r 3}+0.948869 \sigma_{\mathrm{I}}^{r 4}, \\
& \sigma_{\mathrm{II}}^{s}=-1.67085 \sigma_{\mathrm{I}}^{s}-1.51332 \sigma_{\mathrm{I}}^{s 2}+11.0346 \sigma_{\mathrm{I}}^{s 3}+82.8658 \sigma_{\mathrm{I}}^{s 4}+132.917 \sigma_{\mathrm{I}}^{s 5}-91.4241 \sigma_{\mathrm{I}}^{s 6} .
\end{aligned}
$$

The flow law was found to be normal, which implies

$$
\tan \theta=\frac{\dot{\epsilon}_{\mathrm{II}}}{\dot{\epsilon}_{\mathrm{I}}}=-\left(\frac{d \sigma_{\mathrm{II}}^{r, s}}{d \sigma_{\mathrm{I}}^{r, s}}\right)^{-1}
$$

and the stress was calculated from the strain rate direction $\theta$ as the solution of the nonlinear equation

$$
\frac{d \sigma_{I I}^{r, s}}{d \sigma_{I}^{r, s}}=-\cot \theta
$$

The polynomial approximations $\sigma_{I}^{r}(\theta)$ and $\sigma_{I}^{s}(\theta)$ that interpolated our calculated results, and ensure convexity of the yield curves, are
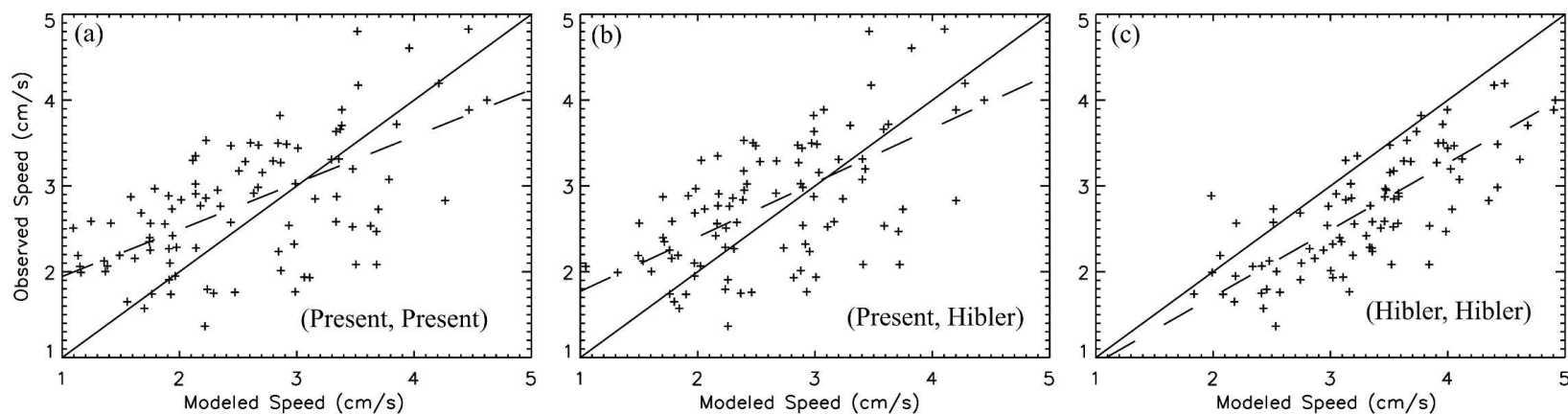

FIG. 17. Scattergrams of the modeled vs Polar Pathfinder-observed monthly mean ice speed for the (a) Present model, (b) Present rheology with Hibler ice strength, and (c) (Hibler, Hibler) model. The dashed line is the linear best fit. 


$$
\begin{aligned}
\sigma_{\mathrm{I}}^{r}= & 2596.89-20003.9 \theta+69046.3 \theta^{2}-140960 \theta^{3}+189140 \theta^{4}-175187 \theta^{5}+114330 \theta^{6}-52593.6 \theta^{7} \\
& +16719.3 \theta^{8}-3499.64 \theta^{9}+434.299 \theta^{10}-24.2182 \theta^{11}
\end{aligned}
$$

for $0.761045 \leq \theta \leq 2.445$,

$$
\sigma_{\mathrm{I}}^{s}=10.633-52.0295 \theta+85.5367 \theta^{2}-47.5057 \theta^{3}
$$

for $0.539315 \leq \theta \leq 0.712814$, and

$$
\begin{aligned}
\sigma_{\mathrm{I}}^{s}= & 60.2245-416.679 \theta+1281.8 \theta^{2}-2321.07 \theta^{3}+2747.66 \theta^{4}-2233.09 \theta^{5}+1272.01 \theta^{6}-508.152 \theta^{7} \\
& +139.623 \theta^{8}-25.15 \theta^{9}+2.6751 \theta^{10}-0.127394 \theta^{11}
\end{aligned}
$$

for $0.712814 \leq \theta \leq 3.01756$. As the yield curves make acute angles with the axis $\sigma_{\mathrm{I}}$ (Fig. 2), the stress values at the two ends of any of the curves (pure divergence and pure convergence) correspond to two ranges of $\theta$ : from 0 to the angle of the normal at the right end (maximum $\sigma_{\mathrm{I}}$ ), and from the angle of the normal to $\pi$ at the left end (minimum $\sigma_{\mathrm{I}}$ ).

\section{REFERENCES}

Armstrong, A., L. B. Tremblay, and L. A. Mysak, 2003: A datamodel intercomparison study of arctic sea-ice variability. Climate Dyn., 20, 465-476.

Bratchie, I., 1984: Rheology of an ice floe field. Ann. Glaciol., 5, 23-28.

Comiso, J. C., P. Wadhams, W. B. Krabill, R. N. Swift, J. P. Crawford, and W. B. Tucker III, 1991: Top/bottom multisensor remote sensing of arctic sea ice. J. Geophys. Res., 96, 2693 2709.

Coon, M. D., 1972: Mechanical behavior of compacted arctic ice floes. Proc. Offshore Technology Conf., Houston, TX, American Institute of Metalurgical, Mining and Petroleum Engineers, 603-608.

Flato, G. M., and W. D. Hibler III, 1995: Ridging and strength in modeling the thickness distribution of arctic sea ice. J. Geophys. Res., 100, 18 611-18 626.

Fowler, C., 2003: Polar Pathfinder daily $25 \mathrm{~km}$ EASE-grid sea ice motion vectors. Tech. Rep., National Snow and Ice Data Center, Boulder, CO, digital media.

Gray, J. M. N., 1999: Loss of hyperbolicity and ill-posedness of the viscous-plastic sea ice rheology in uniaxial divergent flow. $J$. Phys. Oceanogr., 29, 2920-2929.

Heil, P., and W. D. Hibler III, 2002: Modeling the high-frequency component of Arctic sea ice drift and deformation. J. Phys. Oceanogr., 32, 3039-3057.

Hibler, W. D., III, 1979: A dynamic thermodynamic sea ice model. J. Phys. Oceanogr., 9, 815-846.

—_, 1980: Modeling a variable thickness sea ice cover. Mon. Wea. Rev., 108, 1943-1973.

_ , and E. M. Schulson, 1997: On modeling sea-ice fracture and flow in numerical investigation of climate. Ann. Glaciol., 25, 26-32.

— , and — 2000: On modeling the anisotropic failure and flow of flawed sea ice. J. Geophys. Res., 105, 17 105-17 120.

Hilmer, M., and P. Lemke, 2000: On the decrease of Arctic sea ice volume. Geophys. Res. Lett., 27, 3751-3754.
Holland, D. M., 2001: An impact of subgrid-scale ice-ocean dynamics on sea-ice cover. J. Climate, 14, 1584-1601.

Hopkins, M. A., 1994: On the ridging of intact lead ice. J. Geophys. Res., 99, 16 351-16 360.

- 1996: On the mesoscale interaction of lead ice and floes. $J$. Geophys. Res., 101, 18 315-18 326.

- 1998: Four stages of pressure ridging. J. Geophys. Res., 103, $21883-21891$

Hunke, E. C., and J. K. Dukowicz, 1997: An elastic-viscousplastic model for sea ice dynamics. J. Phys. Oceanogr., 27, 1849-1867.

_ , and W. H. Lipscomb, 2001: CICE: The Los Alamos sea ice model. Documentation and software user's manual. Tech. Rep. LA-CC-98-16, Los Alamos National Laboratory, Los Alamos, NM, 56 pp.

Kundu, P. K., 1976: Ekman veering observed near the ocean bottom. J. Phys. Oceanogr., 6, 238-242.

Laxon, S., N. Peacock, and D. Smith, 2003: High interannual variability of sea ice thickness in the Arctic region. Nature, 425, 947-950.

Lewis, J. K., and J. A. Richter-Menge, 1998: Motion-induced stresses in pack ice. J. Geophys. Res., 103, 21 831-21 843.

Miller, P. A., S. W. Laxon, and D. L. Feltham, 2005: Improving the spatial distribution of modeled Arctic sea ice thickness. Geophys. Res. Lett., 32, L18503, doi:10.1029/2005GL023622.

,,,--- and D. J. Cresswell, 2006: Optimization of a sea ice model using basinwide observations of Arctic sea ice thickness, extent, and velocity. J. Climate, 19, 1089-1108.

Moritz, R., and J. Ukita, 2000: Geometry and the deformation of pack ice: I. A simple kinematic model. Ann. Glaciol., 31, 313-322.

Overland, J. E., and C. H. Pease, 1988: Modeling ice dynamics of coastal seas. J. Geophys. Res., 93, 15 619-15 637.

Pritchard, R. S., 1988: Mathematical characteristics of sea ice dynamics models. J. Geophys. Res., 93, 15 609-15 618.

Richter-Menge, J. A., S. L. McNutt, J. E. Overland, and R. Kwok, 2002: Relating Arctic pack ice stress and deformation under winter conditions. J. Geophys. Res., 107, 8040, doi:10.1029/ 2000JC000477.

Rothrock, D. A., 1975: The energetics of the plastic deformation of pack ice by ridging. J. Geophys. Res., 80, 4514-4519.

_ J. Zhang, and Y. Yu, 2003: The Arctic ice thickness anomaly of the 1990s: A consistent view from observations and models. J. Geophys. Res., 108, 3083, doi:10.1029/2001JC001208.

Schulson, E. M., and O. Y. Nickolayev, 1995: Failure of columnar saline ice under biaxial compression: Failure envelopes and 
the brittle-to-ductile transition. J. Geophys. Res., 100, 22383 22400 .

Shen, H. H., W. D. Hibler III, and M. Leppranta, 1987: The role of floe collision in sea ice rheology. J. Geophys. Res., 92, 7085-7096.

Sisodiya, R. G., and K. D. Vaudery, 1981: Beaufort Sea first-year ice features survey-1979. Proc. Sixth Int. Conf. on Port and Ocean Engineering under Arctic Conditions, Quebec City, QC, Canada, Université Laval, 755-764.

Thorndike, A. S., D. A. Rothrock, G. A. Maykut, and R. Colony, 1975: The thickness distribution of sea ice. J. Geophys. Res., 80, 4501-4513.

Tremblay, B. L., and L. A. Mysak, 1997: Modeling sea ice cover as a granular material including the dilatancy effect. J. Phys. Oceanogr., 27, 2342-2360.
Ukita, J., and R. E. Moritz, 1995: Yield curves and flow rules of pack ice. J. Geophys. Res., 100, 4545-4557.

and - 2000: Geometry and the deformation of pack ice: II. Simulation with a random isotropic model and implementation in sea-ice rheology. Ann. Glaciol., 31, 323-326.

Wadhams, P., 1987: Sea ice thickness distribution in the Greenland Sea and Eurasian basin, May 1987. J. Geophys. Res., 97, 5331-5348.

Wilchinsky, A. V., and D. L. Feltham, 2004: Dependence of sea ice yield-curve shape on ice thickness. J. Phys. Oceanogr., 34, 2852-2856.

Zhang, J., D. Rothrock, and M. Steele, 1998: Warming of the Arctic Ocean by a strengthened Atlantic inflow: Model results. Geophys. Res. Lett., 25, 1745-1748. 